\title{
Research Paper \\ Investigating Environmental Barriers Affecting Participation in Patient With Multiple Sclerosis
}

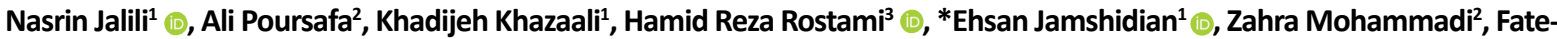 \\ meh Kamali², Nasrin Bahrani ${ }^{2}$
}

1. MSc. Occupational Therapy, Musculoskeletal Research Center, Isfahan University of Medical Sciences, Isfahan, Iran.

2. BSc. Occupational Therapy, Musculoskeletal Research Center, Isfahan University of Medical Sciences, Isfahan, Iran.

3. PhD. Occupational Therapy, Faculty Member of Department of Occupational Therapy, Isfahan University of Medical Sciences, Isfahan, Iran.

\begin{tabular}{|c|c|}
\hline \multicolumn{2}{|r|}{ ( } \\
\hline 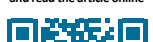 & Citation Jalili N, Poursafa A, Khazaali Kh, Rostami HR, Jamshidian E, Mohammadi Z, Kamali F, Bahrani N. [Investigating \\
\hline 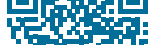 & Environmental Barriers Affecting Participation in Patient With Multiple Sclerosis (Persian)]. Archives of Rehabilitation. 2020; \\
\hline$\Rightarrow+4$ & 21(1):2-21. https://doi.org/10.32598/RJ.21.1.1627.2 \\
\hline 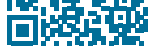 & doil https://doi.org/10.32598/RJ.21.1.1627.2 \\
\hline
\end{tabular}

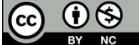

Received: 31 Dec 2018 Accepted: 10 Jul 2019 Available Online: 01 Apr 2020

Keywords:

Environmental barriers, Multiple Sclerosis, Participation

\begin{abstract}
Objective The participation, Based on International Classification of Functioning (ICF), Disability And Health, is part of healthy function that leads to the development of skills and a sense of competence and confidence in them. People with multiple sclerosis experience problems in various areas of participation. Environmental factors can create serious restrictions in the level of participation of people with multiple sclerosis, in some cases, the effect of these factors on restricting the participation of people with multiple sclerosis can be more than the effects of weakness and the defects of systems and organs that result from the disease. the barriers in the physical environment can reduce mobility of people with multiple sclerosis to transfer, that leads consequences such as weakening functional status, physical and mental health, quality of life, happiness, loss of life satisfaction; Therefore, due to high prevalence of multiple sclerosis in the city of Isfahan, the aim of this study was to Identifying the environmental barriers affecting the participation of people with multiple sclerosis in Isfahan.

Materials \& Methods In this cross-sectional study, the study population was all patients with multiple sclerosis in Isfahan City in February 2018. That was conducted in Isfahan province, 96 patients with MS (22.9\%male and $77.1 \%$ female) were selected with convenience sampling method. To collect the data, after informing the participants about the subject and purpose of the study, obtaining informed consent letter, and also ensuring confidentiality of the information to the researcher, a demographic questionnaire was completed. The Mini Mental State Examination questionnaire was completed by participants, and then the Expanded Disability Status Scale scores were recorded for each person by the neurologist. At the end, Craig hospital inventory of environmental factors were provided to the participants. Finally, Data were analyzed by SPSS V. 16.

In order to describe the data, the mean and standard deviation were used. The normal distribution of data was done by Shapiro-Wilk test. Pearson correlation coefficient, ANOVA and post hoc by LSD method test were used to analyze the data at a significance level of 0.05 . Also, considering that the ANOVA test is susceptible to variance inequality, Levin test was conducted to evaluate the equality of variances.

Results Physical and structural barriers are the major barriers to participation of multiple sclerosis patients (12.05 \pm 8.62 ) and the field of work and school is the minor barrier of participation (2.24 \pm 4.16$)$. Furthermore, the findings Also result showed there is no statistical relationship between the demographic variables (such as age, sex, disability status and cognitive status) and level of confrontation
\end{abstract}

* Corresponding Author:

Ehsan Jamshidian, MSc.

Address: Musculoskeletal Research Center, Isfahan University of Medical Sciences, Isfahan, Iran.

Tel: +98 (913) 4331783

E-Mail: jamshidian.ot@gmail.com 
with the environmental barriers of participation $(P>0.05)$. Comparison of the mean of confrontation to environmental barriers to participation Based on education level showed that there is a significant difference between the services and assistance subscale and different levels of education. The highest mean score was for the under diploma education group and the lowest was for the Bachelor's degree and higher group. There is no significant difference in other subscales of participation.

Conclusion According to this findings among the environmental factors that can potentially affect the participation of patients with MS, field of physical and structural barriers were strongest factor which can restrict their participation in personal and social activities. Physical and structural barriers have been introduced in various studies as the most important limiting factor for participation in people with disabilities, especially those with neurological disorders. Therefore, maintaining the participation of these people in the community is a priority in adopting special measures in the field of removing physical and structural barriers.

\section{Extended Abstract}

\section{Introduction}

M

ultiple Sclerosis (MS) is a disease related to the immune system and affects about 2.5 million people worldwide. This disease can cause problems such as fatigue, sensory impairment, motor system disorders, bladder and bowel dysfunction, cognitive impairment, and depression [1]. MS and its disabilities lead to functional limitations and greatly affect people's daily lives and their ability to participate effectively in the family and society [2-4].

The International Classification of Functioning, Disability, and Health defines participation as a person's involvement in a variety of life situations [5]. Participation in purposeful activities promotes self-confidence and skills and leads to physical, mental, and emotional health $[6,7]$. Studies of people with MS show that they experience problems in different areas of participation and activity $[8,9]$.

Attention to the environment as a factor influencing performance has its roots in the history of science. In The Origin of Species, Darwin proposed that the behavior of living organisms is formed based on the environment in which they live [10]. Since then, researchers in various fields have sought to understand the concepts of environment and health and the relationship between them. Ecological theories later emphasized the effects of the environment on participation and suggested the need to change environmental factors instead of focusing solely on changing people's behavior [11]. Recent models of human performance emphasize the environment as a determining factor in the development of disability and agree that disability cannot be understood without considering the environment [12]. Physical, social, and attitude environment can create serious limitations on the level of participation of people with MS [5].

Lexell et al. mentioned the lack of appropriate social support as the most essential factor in limiting the participation of people with MS [13]. In Ephraim and Law studies, the natural and man-made environment have been regarded as the most crucial environmental barriers $[14,15]$. Liao et al. study showed that the design of the physical environment and buildings and services creates the gravest limitations for stroke patients [16]. Environmental barriers can also be one of the reasons for the limited mobility of disabled people, which in turn leads to their social isolation, difficulty in performing daily activities, not participating in leisure activities, lack of proper use of health care services, and the like [17-21].

Given that the prevalence of MS in Isfahan is high [22,23], it is necessary to consider the participation of these people in various personal and social activities. We should identify environmental barriers for their participation and remove them to maintain the independence of these people and increase their quality of life [24]. Using the Craig hospital environmental factors questionnaire, we studied the environmental barriers affecting the participation of people with MS.

\section{Materials and Methods}

The statistical population of the present study consisted of all patients with MS who referred to MS Clinic of Kashani Hospital in Isfahan City, Iran. The sample size for analysis of variance by GPOWER software was estimated as 66 people taking into account the effect size of 0.4 , the type I error of 0.05 , and the test power of 0.8 . Also, the required sample size was estimated to be 84.5 people, taking into account the $r$ value of 0.3 , the type I error of 0.05 , and the test power of 0.8 . 
Table 1. Demographic characteristics of people with Multiple Sclerosis (MS)

\begin{tabular}{|c|c|c|c|c|c|}
\hline Variables & Min & Max & Mean & Std. Err & SD \\
\hline & 25 & 55 & 37.46 & 0.843 & 8.259 \\
\hline & 1 & 22 & 7.36 & 0.507 & 4.972 \\
\hline EDSS & 0 & 6.5 & 2.266 & 0.1946 & 1.907 \\
\hline MMSE & 11 & 30 & 25.46 & 0.38 & 3.725 \\
\hline
\end{tabular}

Table 2. Scores of descriptive information about environmental barrier of participation in Craig Hospital Environmental BarriersQuestionnaire

\begin{tabular}{|c|c|c|}
\hline Variables & Mean \pm SD & $\%$ \\
\hline Policies & $6.866 \pm 6.13$ & \multirow{5}{*}{72.9} \\
\hline Social service & $2.277 \pm 1.9$ & \\
\hline Business policy & $2.769 \pm 2.60$ & \\
\hline Educational policies & $2.403 \pm 1.67$ & \\
\hline Government policies & $2.73 \pm 1.89$ & \\
\hline Physical and structural barriers & $21.05 \pm 8.621$ & \multirow{7}{*}{90.6} \\
\hline Home design & $2.64 \pm 2.17$ & \\
\hline School design & $2.318 \pm 1.5$ & \\
\hline Community design & $2.446 \pm 1.92$ & \\
\hline natural environment & $3.45 \pm 2.917$ & \\
\hline Around & $3.14 \pm 2.998$ & \\
\hline Technology & $2.138 \pm 1.19$ & \\
\hline Work and school & $4.162 \pm 2.24$ & \multirow{4}{*}{40.6} \\
\hline Help at school/workplace & $2.594 \pm 1.81$ & \\
\hline Attitude at school/workplace & $1.833 \pm 1.12$ & \\
\hline Support at school/workplace & $1.64 \pm 0.96$ & \\
\hline Attitudes and support & $8.51 \pm 8.316$ & \multirow{6}{*}{79.2} \\
\hline Attitude at home & $3.04 \pm 2.45$ & \\
\hline Attitude in society & $2.159 \pm 1.51$ & \\
\hline Home support & $2.693 \pm 1.82$ & \\
\hline Community support & $1.952 \pm 1.26$ & \\
\hline Discrimination & $2.616 \pm 2.11$ & \\
\hline Services and assistance & $11.95 \pm 9.442$ & \multirow{8}{*}{86.5} \\
\hline Transportation system & $3.011 \pm 2.86$ & \\
\hline Information & $2.258 \pm 1.7$ & \\
\hline Education & $2.386 \pm 1.79$ & \\
\hline Health Care & $1.988 \pm 1.6$ & \\
\hline Personal equipment & $2.488 \pm 1.39$ & \\
\hline Help at home & $2.761 \pm 2.13$ & \\
\hline Help in the community & $2.386 \pm 1.73$ & \\
\hline Total score & $40.87 \pm 72.797$ & 99 \\
\hline
\end{tabular}


Table 3. Correlation between coping with environmental barriers with age, disease duration, EDSS, and MMSE

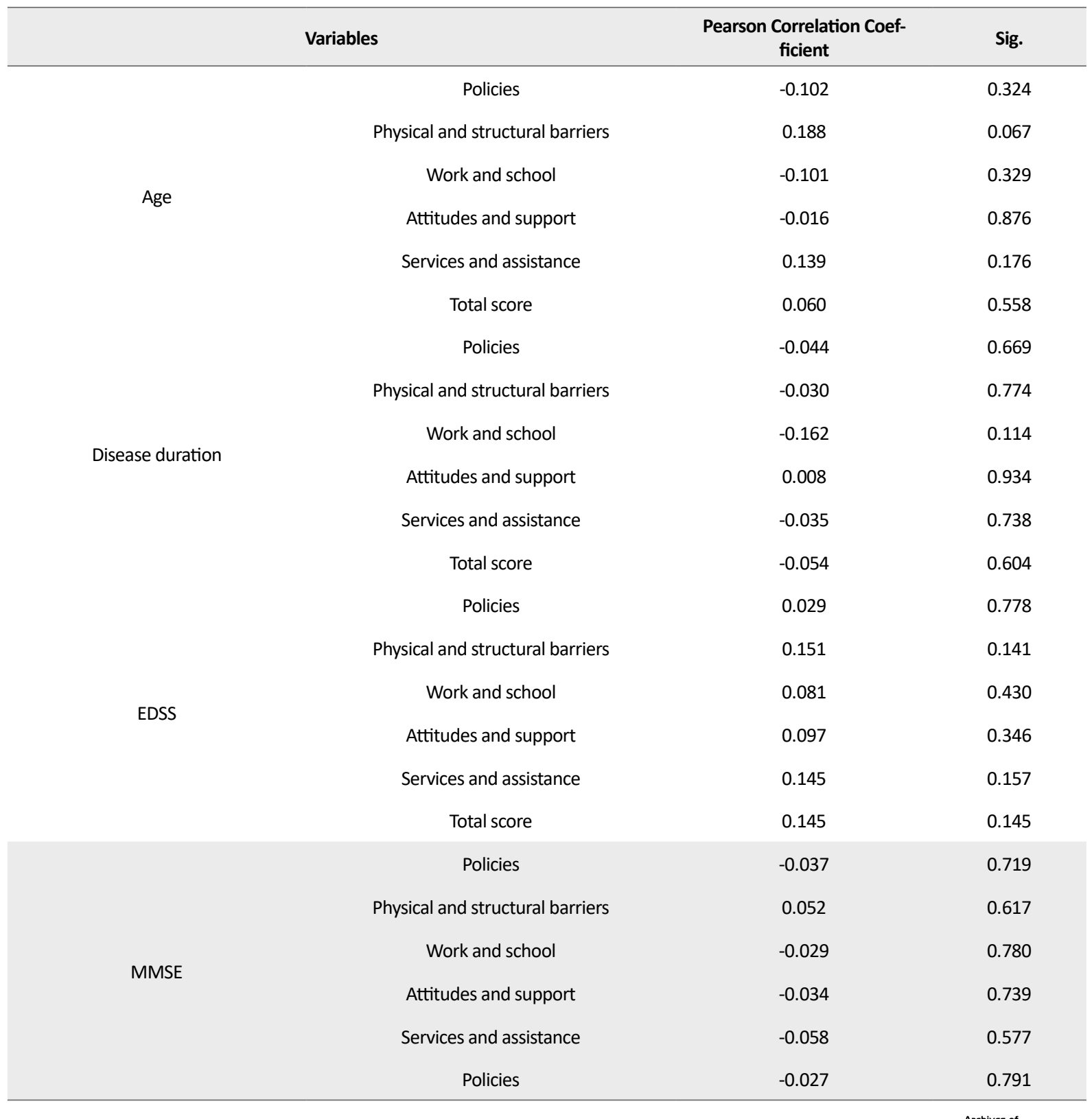

Then, considering the probability of dropout, the sample size was considered to be 100 people. Finally, 96 MS patients were selected by the convenience sampling method. The criteria for admission were 1. diagnosis of MS by a neurologist; 2. age between 25-55 years; 3. living in Isfahan for at least the past year; 4 . not having severe cognitive impairment; 5. consent of the patient to participate in the research.

After informing the participants about the purpose of the research and obtaining their consent, they were assured of the information confidentiality and their demographic information was completed. For each individual, the MiniMental State Examination (MMSE) questionnaire was completed and then the scores on the Expanded Disability Status Scale (EDSS) were recorded by a neurologist. Finally, the Craig Hospital Inventory of Environmental Factors (CHIEF) was provided to the participants to fill it out.

The MMSE test examines orientation, recording information, attention, calculation, recalling, and language skills. The maximum score for this test is 30 . A score between 21 and 26 indicates mild cognitive impairment; a score of 11 to 20 indicates moderate cognitive impairment, and a score of 10 or lower indicates severe cognitive impairment. Using the simultaneous criterion method, the validity of this test was found good. Also, the test reliability was found $78 \%$ 
Table 4. Comparison of the average level of exposure to environmental barriers of participation in terms of education along with the results of analysis of variance

\begin{tabular}{|c|c|c|c|c|c|}
\hline Variables & Statistical Index & No. & Mean $\pm S D$ & $\mathbf{F}$ & Sig. \\
\hline & secondary school and lower & 35 & $4.03 \pm 0.807$ & & \\
\hline \multirow[t]{2}{*}{ Policies } & diploma & 39 & $7.85 \pm 1.276$ & 2.343 & 0.102 \\
\hline & bachelor's degree and higher & 22 & $6.41 \pm 1.487$ & & \\
\hline \multirow{3}{*}{ Physical and structural barriers } & secondary school and lower & 35 & $13.60 \pm 1.703$ & & \\
\hline & diploma & 39 & $11.15 \pm 1.299$ & 1.057 & 0.352 \\
\hline & bachelor's degree and higher & 22 & $11.18 \pm 1.454$ & & \\
\hline \multirow{3}{*}{ Work and school } & secondary school and lower & 35 & $1.77 \pm 0.738$ & & \\
\hline & diploma & 39 & $2.82 \pm 0.672$ & 0.403 & 0.669 \\
\hline & bachelor's degree and higher & 22 & $1.95 \pm 0.812$ & & \\
\hline \multirow{3}{*}{ Attitudes and support } & secondary school and lower & 35 & $8.66 \pm 1.446$ & & \\
\hline & diploma & 39 & $9.95 \pm 1.454$ & 1.927 & 0.151 \\
\hline & bachelor's degree and higher & 22 & $5.73 \pm 1.226$ & & \\
\hline \multirow{3}{*}{ Services and assistance } & secondary school and lower & 35 & $14.09 \pm 1.662$ & & \\
\hline & diploma & 39 & $12.77 \pm 1.480$ & 4.277 & 0.018 \\
\hline & bachelor's degree and higher & 22 & $7.09 \pm 1.633$ & & \\
\hline \multirow{3}{*}{ Total score } & secondary school and lower & 35 & $42.14 \pm 4.905$ & & \\
\hline & diploma & 39 & $44.54 \pm 4.479$ & 1.375 & 0.258 \\
\hline & bachelor's degree and higher & 22 & $32.36 \pm 5.277$ & & \\
\hline
\end{tabular}

Archives of

Table 5. Comparison of the average level of environmental barriers of participation in terms of gender along with the results of the analysis of variance

\begin{tabular}{|c|c|c|c|c|c|}
\hline Variables & Statistical Index & No. & Mean \pm SD & $\mathbf{F}$ & Sig. \\
\hline \multirow{3}{*}{ Policies } & $\operatorname{man}$ & 22 & $8.18 \pm 1.704$ & \multirow{3}{*}{2.604} & \multirow{3}{*}{0.110} \\
\hline & & & & & \\
\hline & woman & 74 & $5.51 \pm 0.747$ & & \\
\hline \multirow{3}{*}{ Physical and structural barriers } & $\operatorname{man}$ & 22 & $2.08010 .95 \pm$ & \multirow{3}{*}{0.460} & \multirow{3}{*}{0.499} \\
\hline & & & & & \\
\hline & woman & 74 & $0.96512 .38 \pm$ & & \\
\hline \multirow{3}{*}{ Work and school } & $\operatorname{man}$ & 22 & $2.36 \pm 0.673$ & \multirow{3}{*}{0.025} & \multirow{3}{*}{0.874} \\
\hline & & & & & \\
\hline & woman & 74 & $2.20 \pm 0.516$ & & \\
\hline \multirow{3}{*}{ Attitudes and support } & man & 22 & $6.55 \pm 1.682$ & \multirow{3}{*}{1.604} & \multirow{3}{*}{0.209} \\
\hline & & & & & \\
\hline & woman & 74 & $9.09 \pm 0.977$ & & \\
\hline \multirow{3}{*}{ Services and assistance } & man & 22 & $1.90511 .55 \pm$ & \multirow{3}{*}{0.510} & \multirow{3}{*}{0.821} \\
\hline & & & & & \\
\hline & woman & 74 & $1.12112 .07 \pm$ & & \\
\hline \multirow{3}{*}{ Total score } & $\operatorname{man}$ & 22 & $6.45139 .59 \pm$ & \multirow{3}{*}{0.060} & \multirow{3}{*}{0.807} \\
\hline & & & & & \\
\hline & woman & 74 & $3.16641 .26 \pm$ & & \\
\hline
\end{tabular}


based on the calculation of the Cronbach $\alpha$. Foroughan et al. have standardized this test in Iran [25].

The EDSS score is a criterion for determining the level of disability of patients with MS and is graded based on the level of neurological damage from 0 (normal neurological status) to 10 (death due to MS) [26].

CHIEF measures the environmental factors that limit participation. Its Cronbach $\alpha$ was reported as 0.93 and its ICC coefficient 0.93 [7]. The validity and reliability of this questionnaire in Iran were examined by Nobakht et al. and reported its Cronbach $\alpha$ coefficient was found 0.86 and the ICC coefficient for all subscales was more than 0.7 [27].

The obtained data were analyzed in SPSS version 16. The mean and standard deviation indicators were used to describe the data. The Shapiro-Wilk test was used to evaluate the normality of data distribution, and the Pearson correlation coefficient statistical test and variance analysis test and post hoc test by LSD method at 0.05 level were used to analyze the data. Also, considering that the variance analysis test is sensitive to variance heterogeneity, the Levene's test was first performed to evaluate the homogeneity of variances.

\section{Results}

Participants in this study included 96 patients with MS, (22.9\% male, and $77.1 \%$ female). Also, the education of the majority of participants $(40.6 \%)$ was at the diploma level. Table 1 lists other demographic information including age, duration of illness, EDSS score, and MMSE score.

Table 2 reports the rate of encountering environmental barriers in different areas of participation according to descriptive statistics. The results show that the highest obtained average (12.05) is related to the subscale of physical and structural barriers and the lowest average (2.24) belonged to the work and school subscale. The percentage of encountering environmental barriers is also mentioned in this Table. In addition to the overall percentage of encountering environmental barriers, the percentage of encountering environmental barriers to scores of 3 or higher (indicating significant, continuous, and effective exposure to environmental barriers) is also mentioned in this Table similar to Ephraim et al. study [14].

Before the statistical analysis, the normality of the data distribution was assessed by skewness and kurtosis, followed by the Shapiro-Wilk test. The results showed that the data distribution was normal $(\mathrm{P}>0.05)$. The Pearson correlation test was used to investigate the relationship between the rate of encountering environmental barriers to participation in different areas, age, duration of disease, EDSS, and MMSE scores (Table 3). No significant relationship was found between the encountering environmental barriers to participation with these variables $(\mathrm{P}>0.05)$. To compare the average rate of encountering environmental barriers to participation in terms of education, Levene's test was performed first. As it confirmed the assumption of homogeneity of variances for the rate of encountering environmental barriers to participation in different areas in terms of education $(\mathrm{P}>0.05)$, the Analysis of Variance (ANOVA) test was used.

Table 4 shows a comparison of the average rate of encountering environmental barriers to participation in different areas (policies, physical and structural, work and education, attitudes and support and services and assistance) in terms of education along with variance analysis results. It was observed that the average rate of encountering environmental barriers to participation in the field of services and assistance is significantly different between various levels of education. The highest average is related to the lower secondary education (or below) group, and the lowest average to the bachelor's degree (or above) group.

There is no significant difference in other areas. To compare the average difference between the rate of encountering environmental barriers to participation in the field of services and assistance in terms of education, the post hoc test using the LSD method was used. The average difference in the rate of encountering environmental barriers to participation in the field of services and assistance is significant between the lower secondary education (or below) group and the bachelor's degree (or above) group, as well as between the diploma and bachelor's degree (or above) groups.

To compare the average rate of encountering environmental barriers to participation in different areas (policies, physical and structural, work and education, attitudes and support and services and assistance) in terms of gender, we first performed the Levene's test to assess the consistency of variances. As the results of the test $(\mathrm{P}>0.05)$ confirmed the similarity of variances for the rate of encountering environmental barriers to participation in different areas in terms of gender, the ANOVA test was used. Table 5 compares the average rate of encountering environmental barriers to participation in different areas in terms of gender. Based on the ANOVA test results, no significant difference between the averages in terms of gender was observed $(\mathrm{P}>0.05)$.

\section{Discussion}

MS affects participation in personal and social activities, and environmental barriers are among the most important factors that can aggravate this limitation. In this study, 
the highest reported environmental barriers were physi$\mathrm{cal}$ and structural and the lowest ones were the work and school. These results were consistent with the findings of the Ephraim et al. study [14]. Also, Hamed stated that the biggest environmental barriers against MS patients are the physical and structural barriers [28]. Using the IPA questionnaire, Cardol et al. in a study of 18- to 75-yearold people in five different disability groups reported the greatest limitations in the areas of work and education [29]. These results are not in line with our results. In addition to the difference in the questionnaire, the minimum age of the subjects studied ( 25 years), and the low level of their education was also significant. Also, Whiteneck and Ephraim stated that the work and school subscale in the Craig questionnaire measures environmental barriers in people who are studying or working but does not measure barriers to employment or education $[14,30]$.

In terms of attitudes and support, the highest score was related to the attitudes at home. Hamed also stated that one of the most crucial environmental barriers is the attitude of first-degree family members and relatives. This finding is significant because of the family-centered culture of countries such as Iran and Jordan [28]. In general, working conditions and attitudes have been suggested as the most important causes of job loss in these patients [31].

Gray et al. introduced physical barriers and attitudes as environmental factors affecting the lives of people with various disabilities, including MS [32]. Khan and Lexell, in their studies on people with MS, also identified attitudes and social support as the most essential environmental factors (13.5). Also, the results of Keysor et al. study showed that in the long run, the most crucial factor that limits patients' participation in activities is the lack of appropriate social support [33].

Our study showed that demographic variables were unrelated to the rate of encountering environmental barriers to participation. This finding is consistent with the results of Badia et al. study. They stated that disability characteristics (such as the level of disability) did not affect patients' participation in leisure activities, but environmental barriers limit participation in activities [34]. The results of the Yorkston et al. study show that in many areas, the rate of participation of people with MS has nothing to do with the severity of their disability. But if the patient's disability increases, his or her sense of confidence and self-efficacy to participate in activities will reduce, and the patient despite having a sense of self-efficacy may not be able to perform the activity due to environmental barriers [35].
The results of the present study concerning demographic variables are not consistent with the results of Ephraim et al. study. Hamed study also shows that demographic variables (age, level of education, duration of illness) are related to MS patients' rate of encountering environmental barriers [28]. Ephraim et al. stated that amputated patients over the age of 55 had a lower rate of encountering environmental barriers than younger patients due to the greater ability of older people to adapt to their disability or because of lower participation in activities [14]. On the other hand, Hamed stated that in older MS patients, the rate of encountering environmental barriers increases due to the long duration of the disease and exacerbation of physical and cognitive problems [28].

The age range of the participants in Ephraim study was 18 to 84 years, so in the present study to reduce the effects of aging on the results of the study, the age range of 25 to 55 years was considered and this factor could be the reason for the difference in results. Hamed study did not specify the age range of the participants, but given that the average age of those participants and the participants in the present study was almost the same, a different measurement method (using a researcher-made questionnaire) could be the reason for the difference in results.

Also, the results of the present study indicate that gender is not a factor affecting the rate of encountering environmental barriers, which is consistent with the results of the Hamed study [28]. These results are inconsistent with the results of the Ephraim study. He stated that the rate of encountering environmental barriers in the field of physical and structural barriers is lower in women than men. $\mathrm{He}$ argued that women do not have high expectations of themselves for doing physical activities [14]. Miller and Dishon argued that women with MS experience more limitations in physical and emotional functioning [36].

Another result of the present study was that the rate of encountering environmental barriers in the field of services and assistance was significantly different in people with different levels of education so that people with a bachelor's degree or higher were less likely to face environmental barriers in this area. Although there are no specific studies on this subject, studies have been conducted on the level of education and employment of MS patients. Honarmand found that lower education did not result in higher unemployment among MS patients [37]. In contrast, Julian et al. found that people with higher levels of education were less likely to lose their jobs [38].

It seems that having a higher level of education makes a person more aware of his/her illness and improves his or 
her ability to cope with the challenges and limitations of the disease and can improve the level of participation of the patients $[39,40]$. According to epidemiological studies, Isfahan is one of the areas with medium to high risk of MS in the world, so it is important to pay attention to the participation of these people in society [41, 42].

\section{Conclusion}

People with MS encounter a high rate of environmental barriers, especially in the area of physical and structural barriers. These barriers can limit their participation in personal and social activities.

Because of the high prevalence of MS in Iran and the high rate of exposure of these patients to environmental barriers, it seems necessary to eliminate environmental barriers and provide these patients the possibility of participating in individual and social activities, which can improve their wellbeing and quality of life. It is suggested that in future studies, the rate of encountering environmental barriers be evaluated based on different types of multiple sclerosis. In the field of clinical work, it is also suggested that specialists and therapists, besides considering the limitations of performance and body shape, examine the evaluation and intervention of environmental barriers.

\section{Ethical Considerations}

\section{Compliance with ethical guidelines}

This study ethically approved in Ethics Committee of Isfahan University of Medical Sciences (Code: IR.MUI. REC.1394.2.074)

\section{Funding}

The present article is the result of an approved research project at Isfahan University of Medical Sciences (Code number: 294074).

\section{Authors' contributions}

Conceptualization, supervision: Nasrin Jalili; Methodology: Nasrin Jalili, Ehsan Jamshidian; Investigation, project administration: Nasrin Jalili, Ehsan Jamshidian, Ali Pour Safa; Writing-review \& editing, visualization, funding acquisition: All authors.

\section{Conflicts of interest}

The authors declared no conflict of interest.

\section{Acknowledgements}

The authors of the article would like to express their gratitude to the Center for Musculoskeletal Research of the Faculty of Rehabilitation of Isfahan University of Medical Sciences, who financially supported this research. 
This Page Intentionally Left Blank 


\title{
بررسى موانع محيطى اثر تذار بر مشاركت در افراد مبتلا به مولتييل اسكلروزيس
}

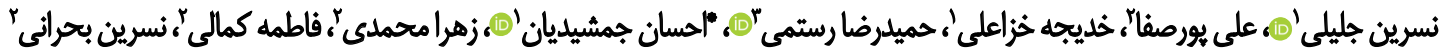

1. كارشناس ارشد كاردرمانى، مركز تحقيقات اختلالات اسكلتى و عضلانى، دانشكاه علوميزشكى اصفهان، اصفهان، ايران.

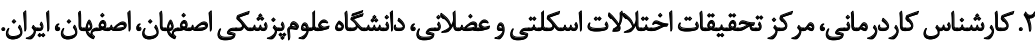

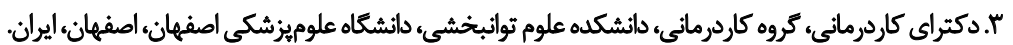

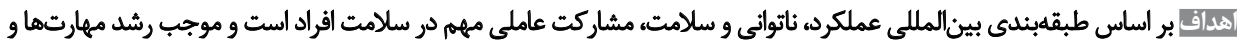

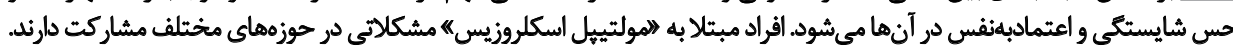

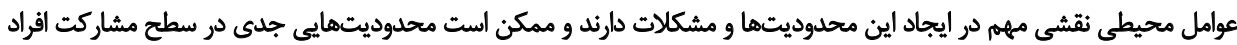

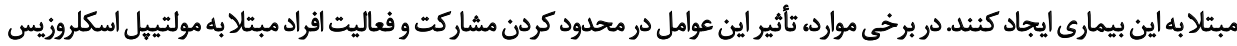

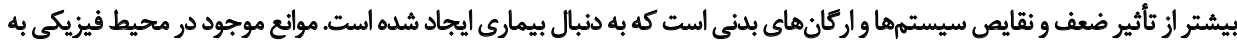

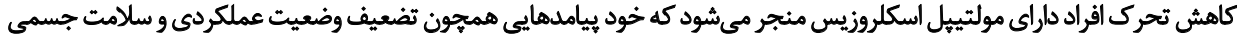

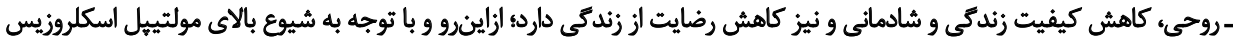

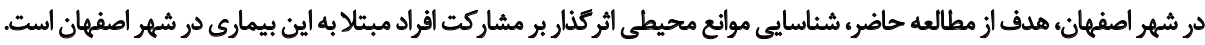

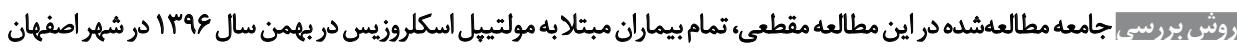

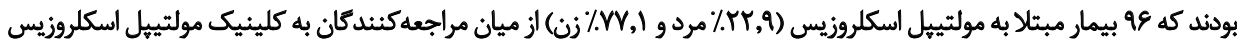

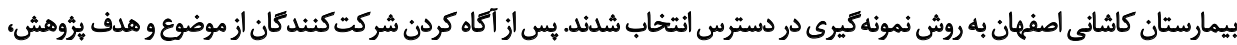

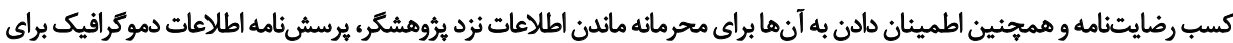

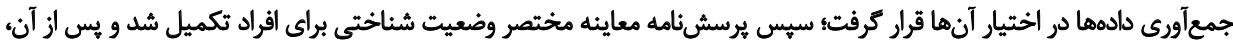

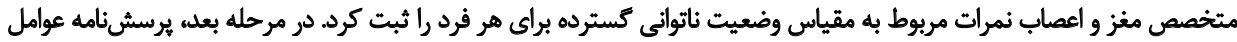

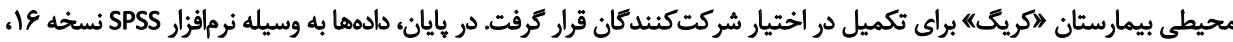

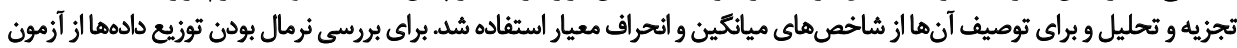

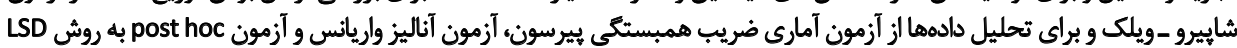

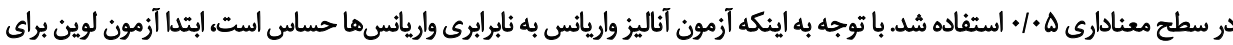

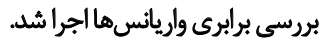

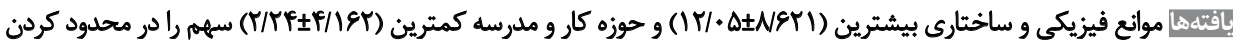

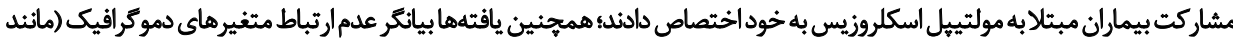

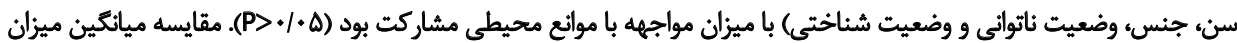

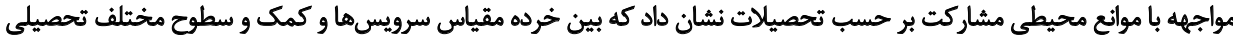

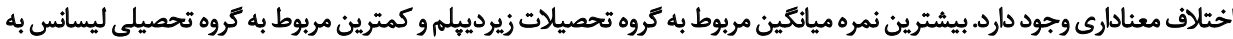

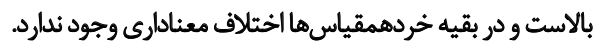

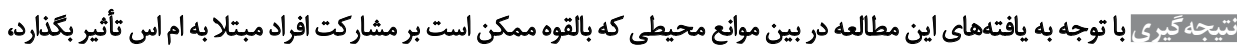

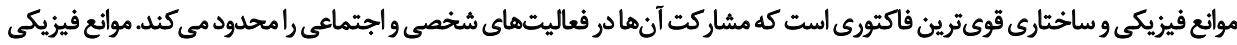

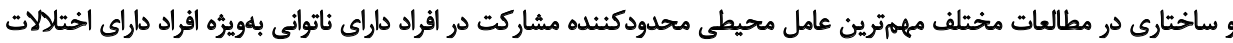

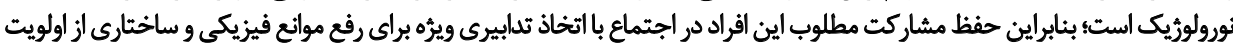
بالايى برخوردار است.

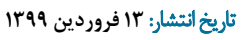

-

كليدوارثهها:

موائع محيطي،

مشاركت، مولتيبل مونيل

اسكلروزيس

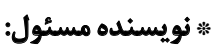

احسان جمشيديان

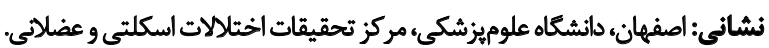

تلفن:

راياثامه: jamshidian.ot@gmail.com
\end{abstract}


است كه اقراد جامعه در آن زندكى مي كنيند. اين عوامل با شرايط

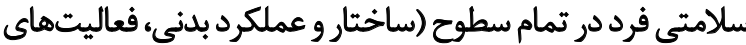

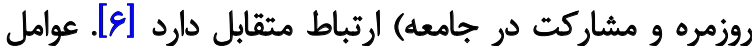

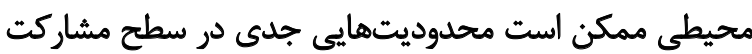

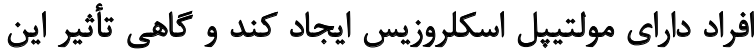

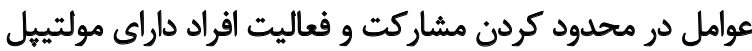

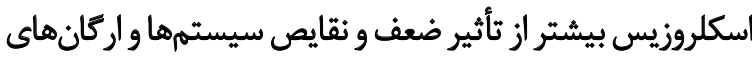

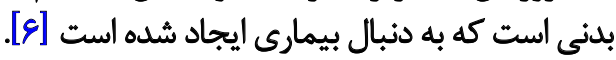

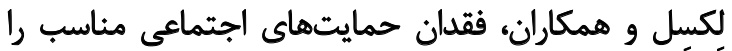

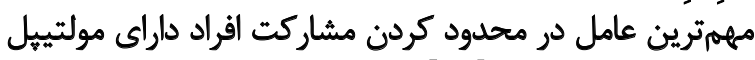

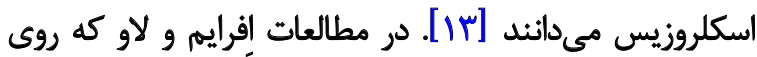

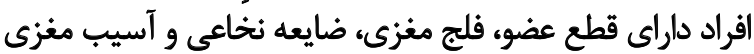

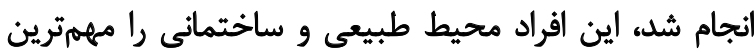

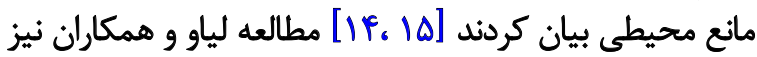

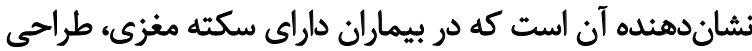

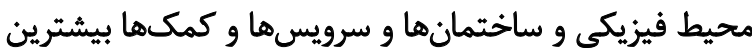

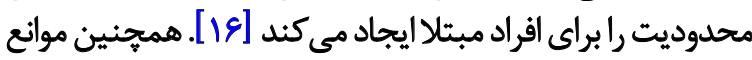

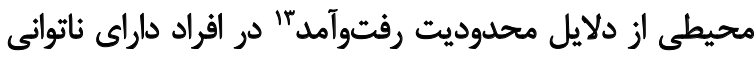

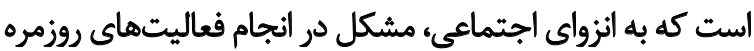

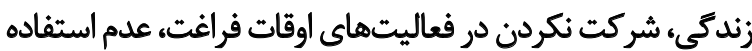

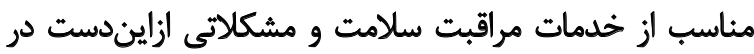

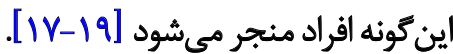

با توجه به اينكه ايران و بهخصوص شهر اصفهان در رده مناطق

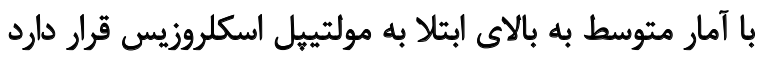

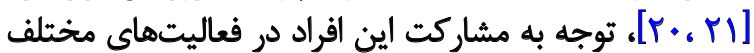

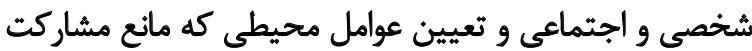

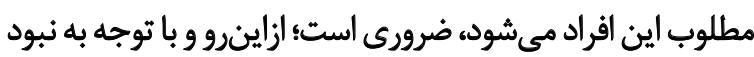

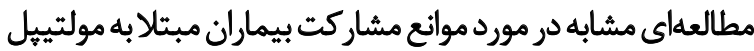

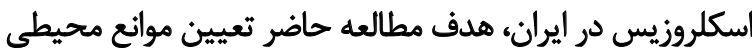
اثركذار بر مشاركت افراد داراي مولتييل اسكلروزيس است.

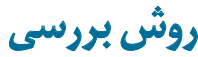

مطالعه حاضر مطالعهاي مقطعى است كه جامعله آمارى آن

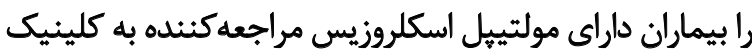

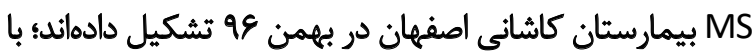

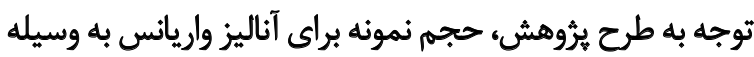

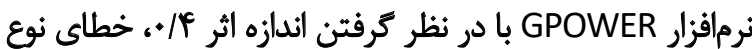

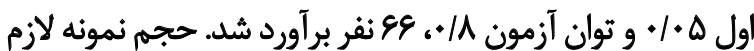

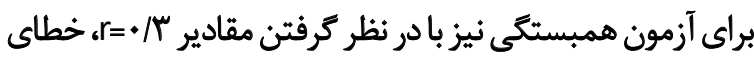

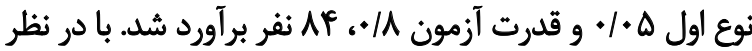

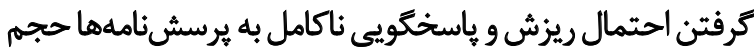

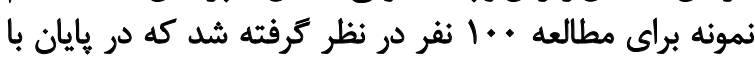

13. Transportation
مقدمه

مولتييل اسكلروزيس' از بيمارىهاى مربوط به سيستم

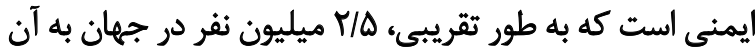

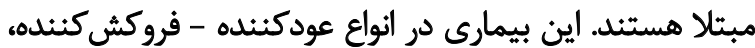

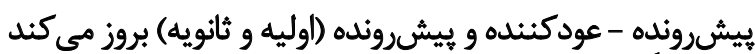

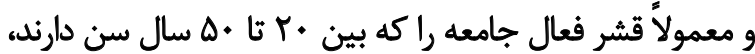

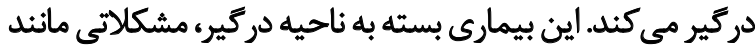

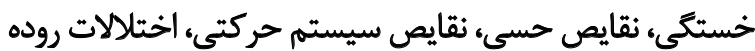

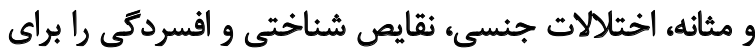

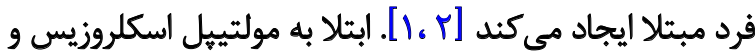

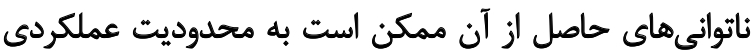

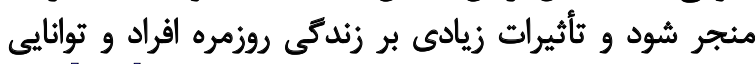

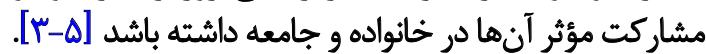

سازمان جهانى بهداشت در طبقلبندى بينالمللى عملكرد،

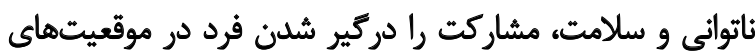

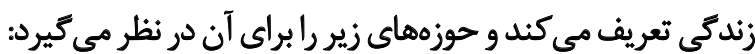

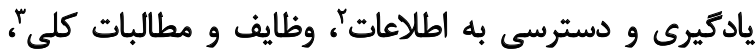

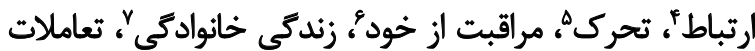

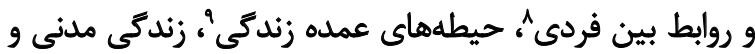

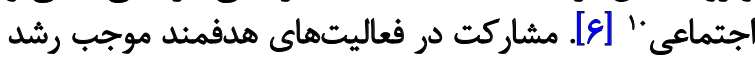

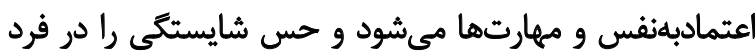

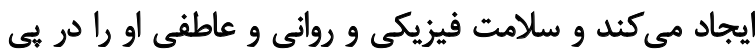

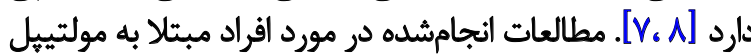

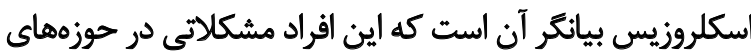

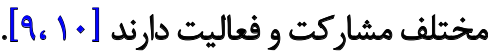

تاثير رويكردهاى كاردرمانى، مدل اكولوريك عملكرد انسان بر بر براز

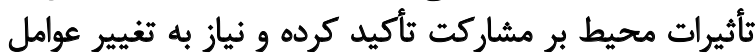

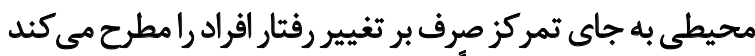

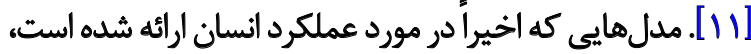

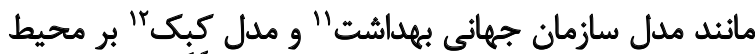

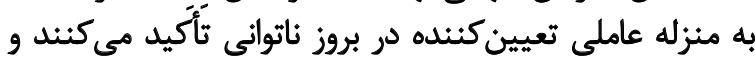

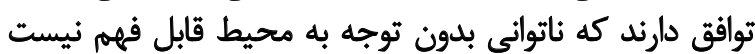

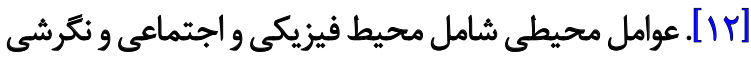

1. Multiple Sclerosis (MS)

2. Learning and applying knowledge

3. General tasks and demands

4. Communication

5. Mobility

6. Self-Care

7. Domestic life

8. Interpersonal interactions and relationships

9. Major life areas

10. Social and civil life

11. International classification of functioning, disability and health (ICF)

12. Quebec 


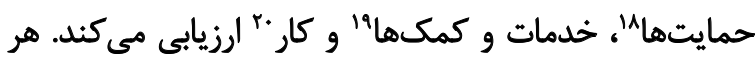

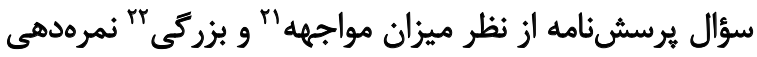

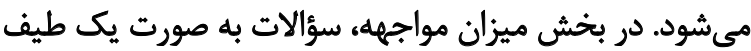

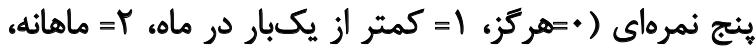

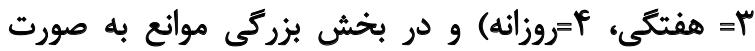

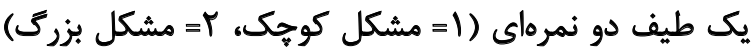

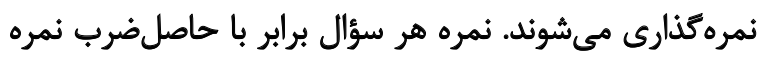

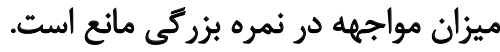

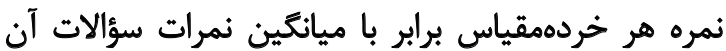

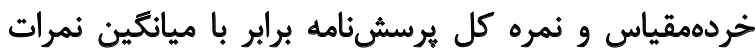

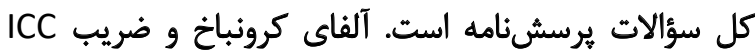

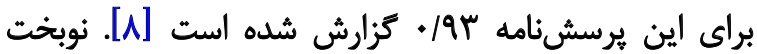

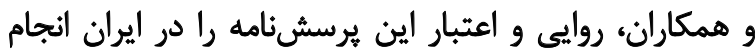

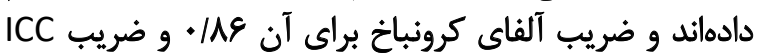

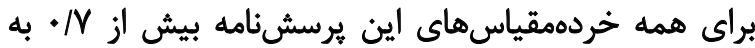

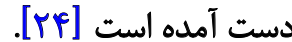

در غايان، دادهها به وسيله نرمافزار SPSS نسخه

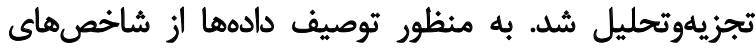

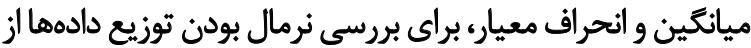

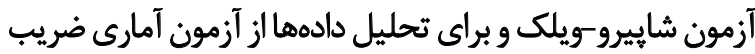

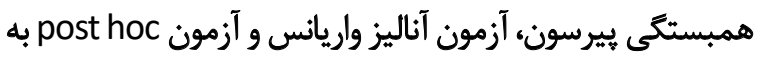

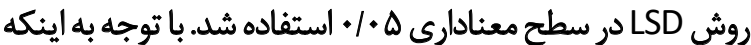

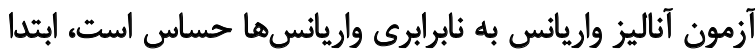

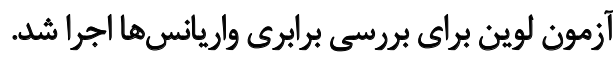

يافتهانها

افراد شركتكنيده در اين مطالعه צو بيمار داراي مولتيخيل

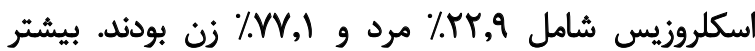

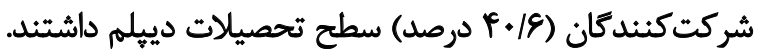

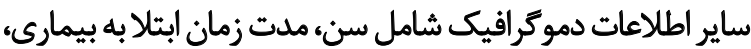
نمره EDSS و نمره MMSE دركافيك جدامل سنول شماره آمآ آمده است. ميزان مواجهه با موانع محيطى مشاركت در حيطههاى

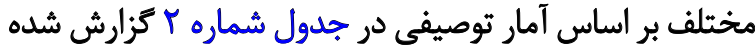

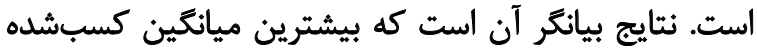

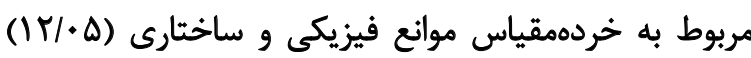

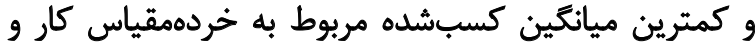

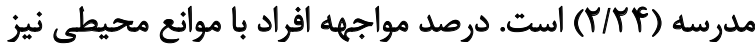
در اين جدول ذكر شده است.

18. Attitudes and Support

19. Services and Assistance

20. Work

21. Frequency

22. Magnitude
توجه به ملاكهاى ورود، و9 بيمار داراى مولتييل اسكلروزيس

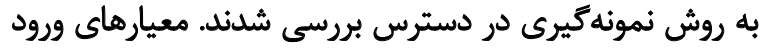

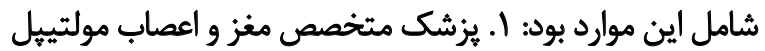

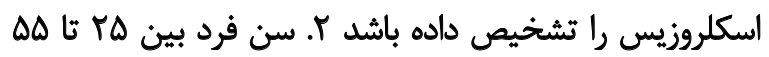

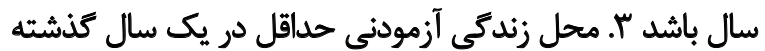

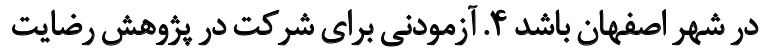
داشته باشد ه. سواد خواندن و نوشتن داشته باشئ باشد.

ملاك خروج از مطالعه نيز اختلال شناختى شديد (نمره زير

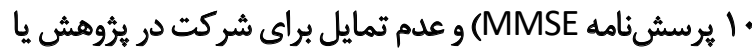
تمايل به ادامه همكارى در طرح حاضر بود.

يس از آكاه كردن شركت كنند كان از موضوع و هدف يرّوهش،

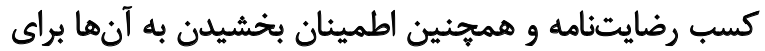

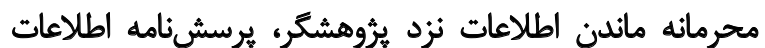

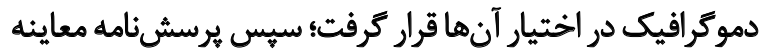

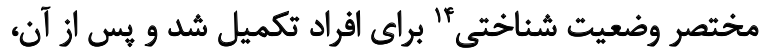

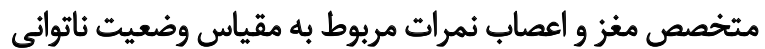

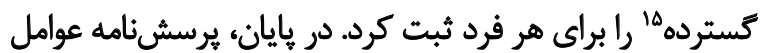

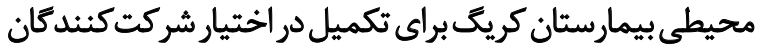

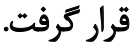

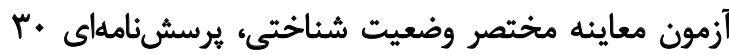

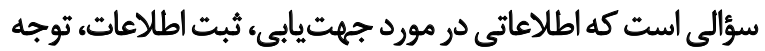

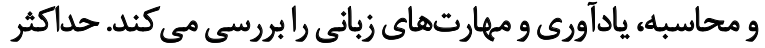

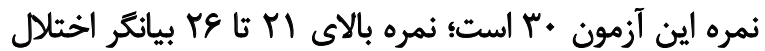

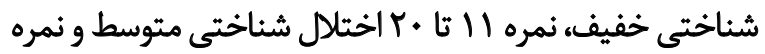

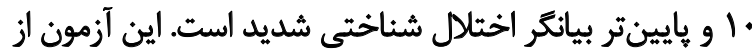

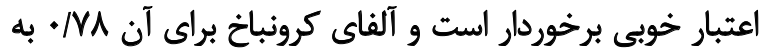

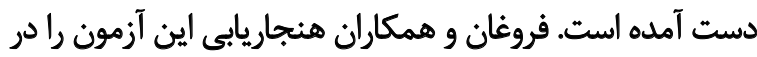

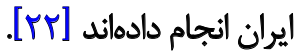

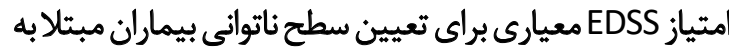

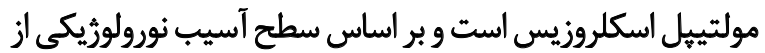

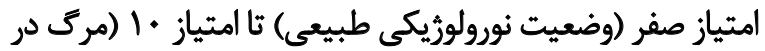

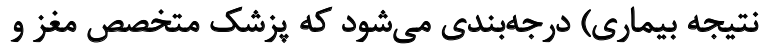

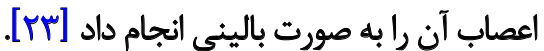

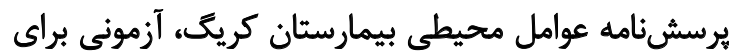

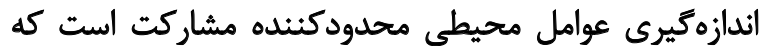

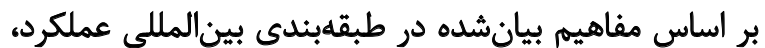

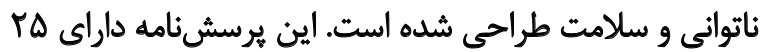

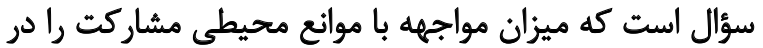

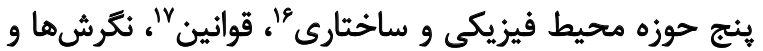

14. Mini Mental State Examination (MMSE)

15. Expanded Disability Status Scale (EDSS)

16. Physical and Structural

17. Policies 


\begin{tabular}{|c|c|c|c|c|c|}
\hline اتحراف معيار & خطاى استاندارد & مياتكين & بيشترين & كمترين & متغير \\
\hline NTAQ & /ART & rVles & $\Delta \Delta$ & ro & سن \\
\hline r/arr & $\cdot / \Delta \cdot Y$ & V/re & r & 1 & طول مدت بيمارى (سال) \\
\hline $1 / 9 \cdot r$ & ./1948 & T/REg & $9 / \Delta$ & - & EDSS \\
\hline$T / M O$ &.$/ \mu \lambda$ & TA/ศE & $r$ & 11 & MMSE \\
\hline
\end{tabular}

جدول ץ. اطلاعات توصيفي نمرات مواجهه با موائ محيطى مشاركت در برسشئامه موانع محيطي بيمارستان كريقَ

\begin{tabular}{|c|c|c|}
\hline درصد & ميانكين土|نحراف معيار & 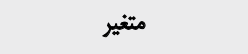 \\
\hline \multirow{5}{*}{$\pi / 9$} & EINEFISIN & سياستها \\
\hline & $r / T r \pm) / q$ & خلمات اجتماعى \\
\hline & $T / N E q \pm T / .8$ & سياسيت مشاغل \\
\hline & $r / x+r \pm V / Q V$ & سياستهاى آموزشى \\
\hline & $r / M^{\prime} \pm V / A q$ & سياستهاي دولثى \\
\hline \multirow{7}{*}{9.18} & $\mid Y / . Q \pm N E Y I$ & موانع فيزيكى و ساختارى \\
\hline & $r / \mathscr{T P} \pm \mathrm{Y} / \mathrm{IV}$ & طراحى خانه \\
\hline & $r / M U \pm N / \Delta$ & طراحى هلرسه \\
\hline & r/PFe \pm I/QT & طراحى جامعه \\
\hline & $r / r \Delta \pm r / q I V$ & محيط طبيعى \\
\hline & $r / / F \pm Y / 4$ Q & ييرامون \\
\hline & $r / 1 r A \pm V / / q$ & 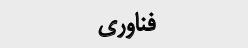 \\
\hline \multirow{4}{*}{$r+18$} & F/IETET/MF & كار و ملدرنسه \\
\hline & V/OQTEI/A) & كمى در ملرسه/ /محل كار \\
\hline & VArTI $\pm / M r$ & نكرش در ملرسه/محل كار \\
\hline & $V / q F \pm \cdot / q$ & حمايت در ملرسه/محل كار \\
\hline \multirow{6}{*}{$v a / r$} & NAI \pm NTIS & نكرشها و حمايت \\
\hline & $r / P \pm Y / P Q$ & نكرش در خانه \\
\hline & $r /(\Delta q \pm V / \Delta)$ & نكرش در اجتماع \\
\hline & r/gar \pm V/AT & حمايت در خائه \\
\hline & VARt)/E & حمايت در اجتماع \\
\hline & $r / \& \mid \& \pm T / U I$ & تبعيض \\
\hline \multirow{8}{*}{$N / \Delta$} & W/RADa/PFT & سرويسها و كمى \\
\hline & $r / .11 \pm r / N$ & سيسته حملونقل \\
\hline & $r / T A M \pm N / Y$ & أطلاعات \\
\hline & T/TAEIN/M & أموزش و تربيت \\
\hline & $1 / q u \pm 1 / 8$ & مراقبتهاى يزٔشكى \\
\hline & $r / T M \pm V / T q$ & تجهييزات شخصصى \\
\hline & TMEIET/N & كمى در خانه \\
\hline & $T / M N E D / M$ & كمك در جامعه \\
\hline 99 & $r \cdot / A Y \pm Y V / V 9 Y$ & نمره كل \\
\hline
\end{tabular}


جدول ب. همبستكى بين ميزان مواجهه با موانع محيطى مشاركت با سن، طول مدت بيمارى، EDSS و MMSE

\begin{tabular}{|c|c|c|c|}
\hline سطح معنادارى & ضريب همبستكي ييرسون & متغير & \\
\hline . /TIY & $-.11 \cdot r$ & سياستها & \multirow{6}{*}{ ن } \\
\hline .1 .98 &.$/ M$ & موانع فيزيكى و ساختارى & \\
\hline + ( & $-+11+1$ & كار و ملرسه & \\
\hline$\cdot / A V E$ & -.1 .18 & نكرشها ورمايت & \\
\hline.$/ 1 V 8$ &.$/ 1 F 9$ & سرويسها وكمى & \\
\hline.$/ \Delta Q A$ & .1 .9$. & نمره كل & \\
\hline .1999 &.$- / .+4$ & سياستها & \multirow{6}{*}{ طول مدت بيمارى } \\
\hline$t / M e$ & $-* /+\%$ & موائع فيزيكى و ساختارى & \\
\hline.$/ 114$ &.$- / 1 E T$ & كار و هدرسه & \\
\hline ./9M & $+10+1$ & نكرشها وحمايت & \\
\hline.$/ M \Lambda$ & -.1 .40 & سرويسها وكمى & \\
\hline $.18+4$ &.$- / .0 p$ & نمره كل & \\
\hline.$/ W A$ & .1 .49 & سياستها & \multirow{6}{*}{ EDSS } \\
\hline.$/ P H$ & $.|| \Delta \mid$ & موانع فيزيكى و ساختّارى & \\
\hline$. / R+$. & $*(* 1)$ & كار و هلرسسه & \\
\hline.$/ \pi q$ & $.1 .9 V$ & نكرشها و حمايت & \\
\hline ./\aV & IIFA & سرويسها وكمى & \\
\hline IIFD & $+/ N A$ & 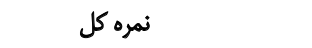 & \\
\hline.$M I 9$ & $-.1 \cdot \mathrm{HV}$ & نسياستها & \multirow{6}{*}{ MMSE } \\
\hline.$/ 818$ & $\%$ H $\Delta r$ & موانع فيزيكى و ساختارى & \\
\hline.$/ Y \Lambda_{\text {. }}$ & -.1 .49 & كار و ملرسيه & \\
\hline.$/ N 9$ & $-.1 \cdot \pi$ & نكرشها و حمايت & \\
\hline.$/ \Delta W$ & $-* 1+\Delta 4$ & سرويسها و كمى & \\
\hline.$/ 291$ & $-.1 \cdot r \gamma$ & نمره كل & \\
\hline
\end{tabular}

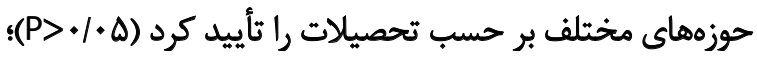

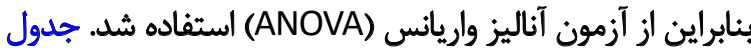

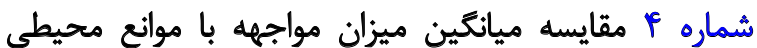

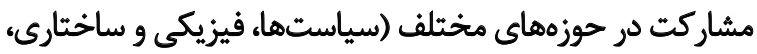

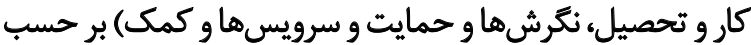

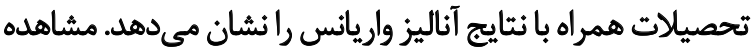

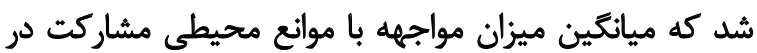

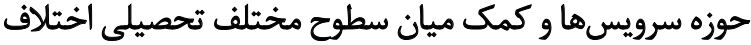

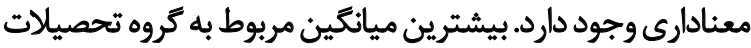

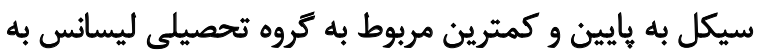

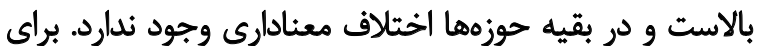

تيش ازي انجام تحليل آمارى، نرمال بودن توزيع دادهها از طريق إزيق

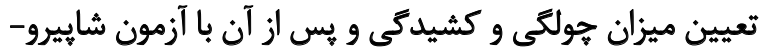

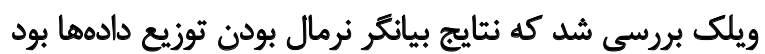

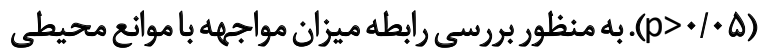

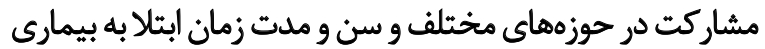

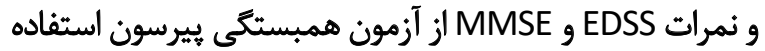

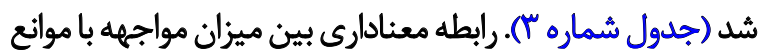

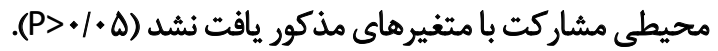
براى مقايسه ميانگين ميزان مواجهه با موانع محيطى مشاركت

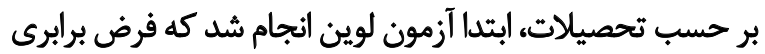

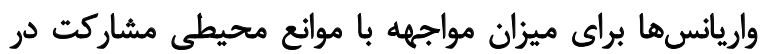


جدول ع. مقايسه ميانكين ميزان مواجهه با موانع محيطى مشاركت برحسب تحصيلات همراه با نتايج آناليز واريانس

\begin{tabular}{|c|c|c|c|c|c|c|}
\hline سطح معنادارى & $\mathbf{F}$ & خُأناي & مياتكين & تعداد & شاخص آمارى & متغير \\
\hline \multirow{3}{*}{$\cdot N \cdot r$} & \multirow{3}{*}{ T/MTM } & $\cdot / \Lambda \cdot \gamma$ & $r /+r$ & ro & سيكل و يايينتر & \multirow{3}{*}{ سياستها: } \\
\hline & & ITVE & V/AS & rq & دييلم & \\
\hline & & I/EAY & $s / 4 \mid$ & rr & ليسانس و بالاتر & \\
\hline \multirow{3}{*}{. THAY } & \multirow{3}{*}{$V \cdot \Delta V$} & $1 / N \cdot r$ & $1 w / 8$. & ra & سيكل و يايينتر & \multirow{3}{*}{ موانع فَيزيكى و ساختارى } \\
\hline & & $1 / 799$ & $11 / 10$ & ma & دييلم & \\
\hline & & $1 / F \Delta F$ & $W / M$ & ir & ليسانس و بالاتر & \\
\hline \multirow{3}{*}{.1899} & \multirow{3}{*}{$\cdot / 4+r$} & . Mra & $1 / W$ & ro & سيكل و يايينتر & \multirow{3}{*}{ كار و ملرسه } \\
\hline & & $.18 V T$ & $r / A T$ & rq & دييلم & \\
\hline & & . IAIr & $1 / 9 \Delta$ & it & ليسانس و بالاتر & \\
\hline \multirow{3}{*}{.$/ 101$} & \multirow{3}{*}{ VarV } & Whes & N\&E & ra & سيكل و يايينتر & \multirow{3}{*}{ تكرشها و حمايت } \\
\hline & & $1 / F \Delta T^{5}$ & $9 / 9 \Delta$ & rq & دييلم & \\
\hline & & MTE & $\Delta M$ & r & ليسانس و بالاتر & \\
\hline \multirow{3}{*}{$.1 \cdot 11$} & \multirow{3}{*}{$F / T W$} & Wer & $1 f / \cdot 9$ & ra & سيكل و بإيينتر & \multirow{3}{*}{ سرويسها وكمى } \\
\hline & & $1 / F A$. & $I T / W$ & rq & دييلم & \\
\hline & & V/RT & $V / \cdot 9$ & rt & ليسانس و بالاتر & \\
\hline \multirow{3}{*}{.$/ T A A$} & \multirow{3}{*}{ I/TVA } & $F / 9.0$ & TT/NF & ra & سيكل و وايينتر & \multirow{3}{*}{ نمره كل } \\
\hline & & e/pra & 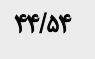 & rq & دييله & \\
\hline & & $\Delta / T W$ & & m & ليسانس و بالاتر & \\
\hline
\end{tabular}

توانبخننى

جدول ه ممقايسه ميانكين ميزان مواجهبه با موانع محيطي مشاركت برحسب جنسيت همراه با نتايج آزمون آناليز واريانس

\begin{tabular}{|c|c|c|c|c|c|c|}
\hline سطح معنادارى & F & خطاى استاندارد & مياكين & تعداد & شاخص آمارى & متغير \\
\hline \multirow{3}{*}{.111} & \multirow{3}{*}{$r / 8 \cdot f$} & W.F & NIM & $\pi$ & مرد & \multirow{3}{*}{ سياستها| } \\
\hline & & & & & & \\
\hline & & - Mr & $\Delta / \Delta 1$ & $n$ & is & \\
\hline \multirow[b]{2}{*}{.$/ 199$} & \multirow[b]{2}{*}{$.158+$} & $r / * A *$ & $1 . / 9 \Delta$ & $\pi$ & مرد & \multirow[b]{2}{*}{ موانع فيزيكى و ساختارى } \\
\hline & & 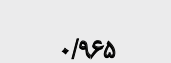 & ג'ז/ & $n^{*}$ & ii & \\
\hline \multirow{3}{*}{.$/ A n^{\circ}$} & \multirow{3}{*}{. $/$ YO } & $.8 n$ & 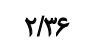 & $\pi$ & هرد & \multirow{3}{*}{ كار و مدرسه } \\
\hline & & & & & & \\
\hline & &.$/ 018$ & $T / T_{0}$ & $n$ & ji & \\
\hline \multirow{3}{*}{$\cdot \pi \cdot 9$} & \multirow{3}{*}{$V / 8 \cdot 4$} & VFAT & g/DA & $\pi$ & مرد & \multirow{3}{*}{ نكرش هاو حمايت } \\
\hline & & & & & & \\
\hline & & . $99 \mathrm{~W}$ & $9 /=9$ & $n^{*}$ & is & \\
\hline \multirow{3}{*}{$\cdot|A M|$} & \multirow{3}{*}{.$/ \Delta 1}$. & $1 / 9.0$ & $1 W / \Delta \Delta$ & $m$ & هرد & \multirow{3}{*}{ سرويسها وكمى } \\
\hline & & & & & & \\
\hline & & V/rI & $\mid r / \cdot V$ & $n$ & j & \\
\hline \multirow{2}{*}{$\cdot / A \cdot V$} & \multirow{2}{*}{.1 .8}$. & SPADI & $r q / \Delta q$ & $\pi$ & مرد & \multirow{2}{*}{ نمرهكل } \\
\hline & & P/NE & FI/Te & $\eta$ & is & \\
\hline
\end{tabular}

توانبخننى 
اتخاذ تدابيرى ويرٔه براى رفع موانع فيزيكى و ساختارى از إز إنائ اولويت بالايى برخوردار است [عادئ.

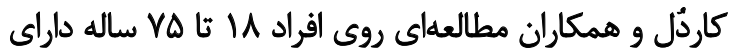

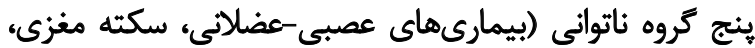

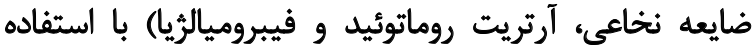

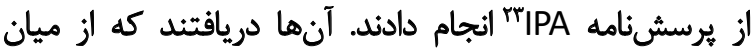

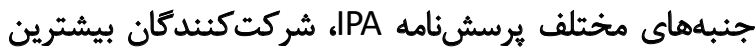

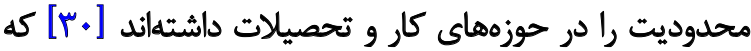

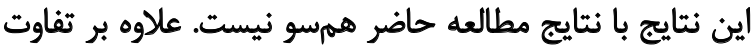

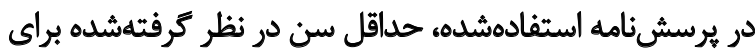

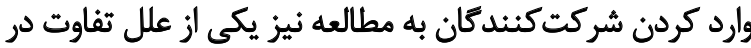

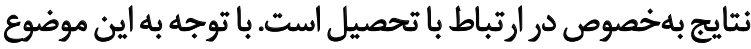

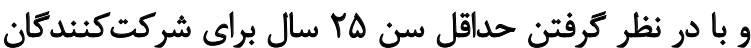

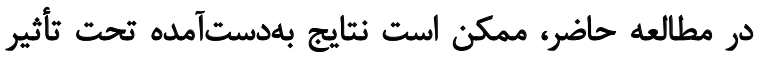

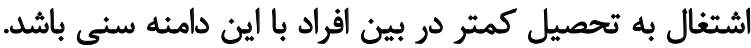

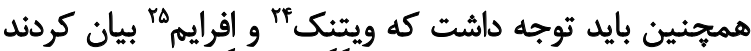

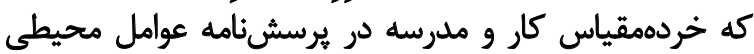

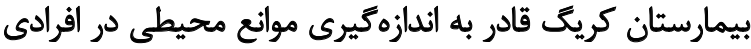

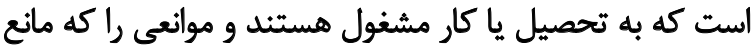

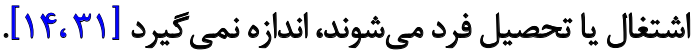
در حوزه نكَرشها و حمايت، در مطالعه حاضر بيشترين نمره

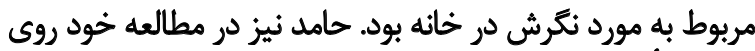

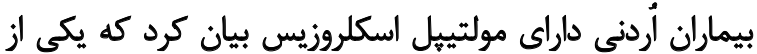

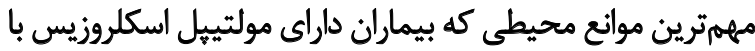

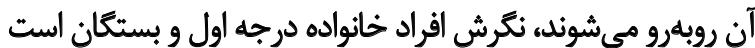

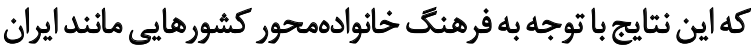

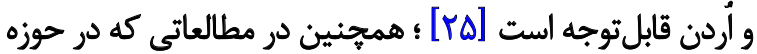

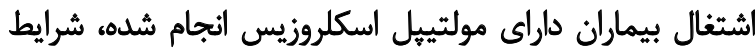

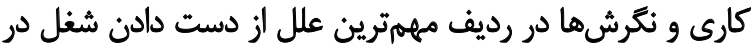

بيماران داراى مولتييل اسكلروزيس مطرح شده است [برت]

كراى و همكاران نيز نترش ها را جزء موانع محيطى تأثيركذار

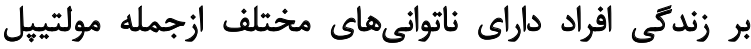

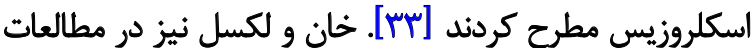

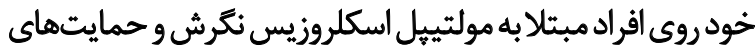

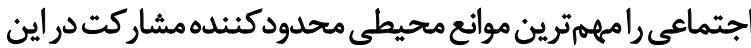

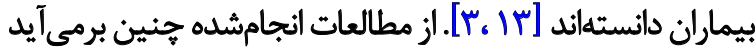

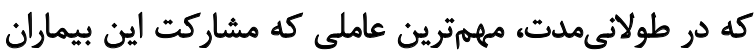

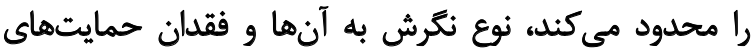

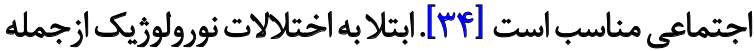
مولتييل اسكلروزيس تأثيرات روانشناختى فراوانى مانند تجربه

23. Impact on Participation and Autonomy questionnaire 24. Whiteneck

25. Ephraim
مقايسه اختلاف ميانكين ميزان مواجهه با موانع محيطي مشاركت

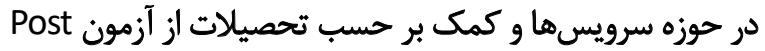

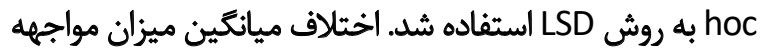

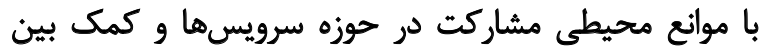

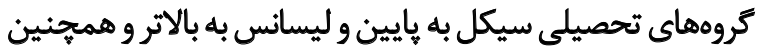
بين گروههاى دييلم و ليسانس به بالاتر معنادار است.

براى مقايسه ميانكين ميزان مواجهه با موانع محيطى

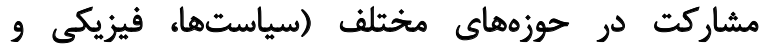

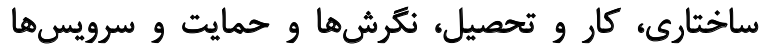

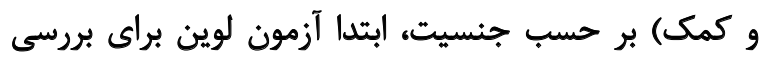

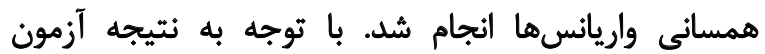

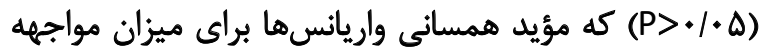

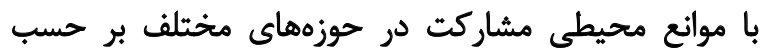

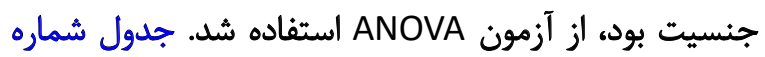
ه مقايسه ميانكين ميزان مواجهه با موانع محيطى مشاركت

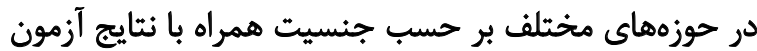

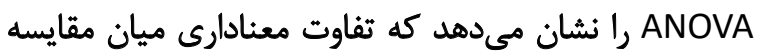

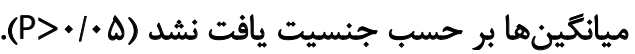

بحث

نتايج بهدستآمده در يُؤوهش حاضر بيانكَ آن است كه

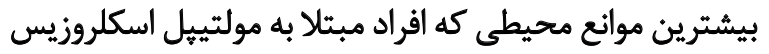

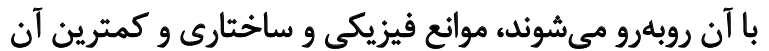
مربوط به حوزه كار و مدرسه است ائ اين نتايج با نتايج مطالئه

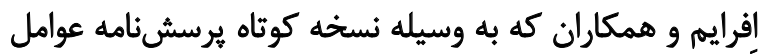

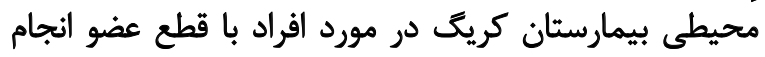

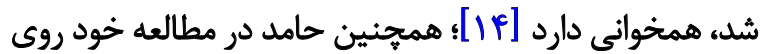

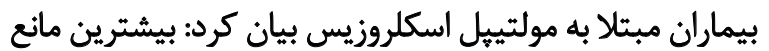

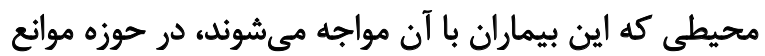

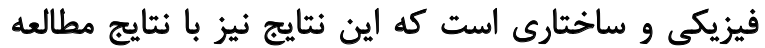

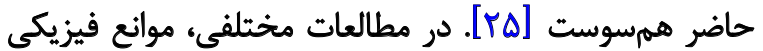

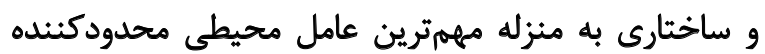

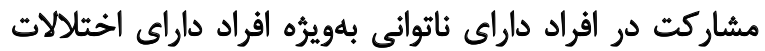

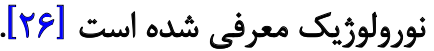
افراد داراى اختلالات نورولوزيك مائند مولتييل اسكلروزيس

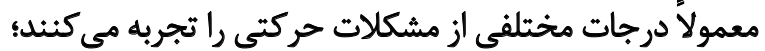

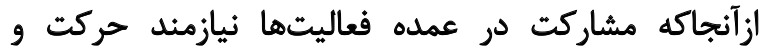

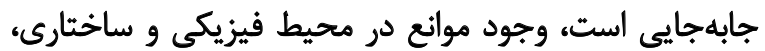

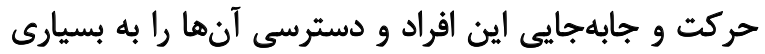

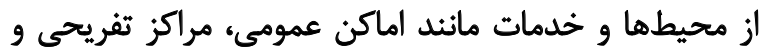

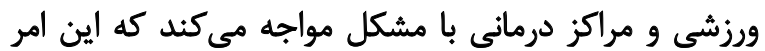

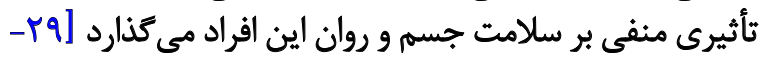
TrV 
يا شركت كمتر آنها در فعاليتها باشد [f [1]]. مطالعه حامد نيز

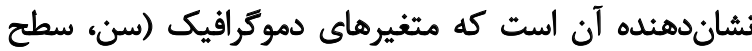

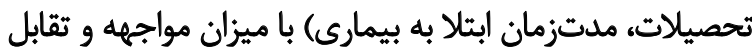

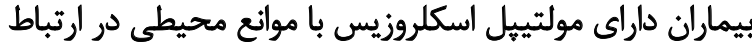

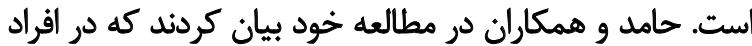

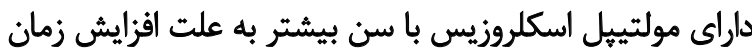

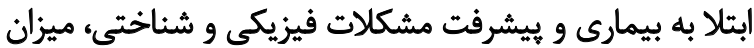

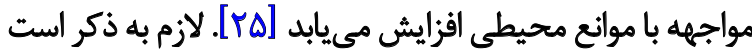

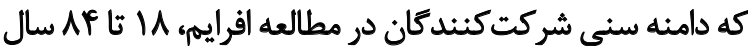

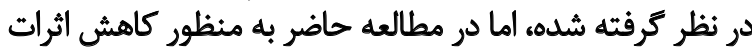

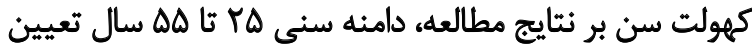

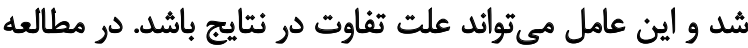

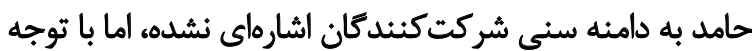

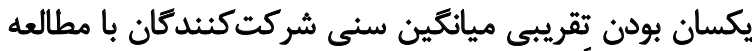

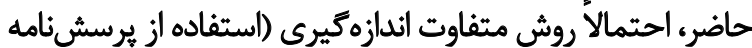
محققساخته) علت تفاوت در نتايج است.

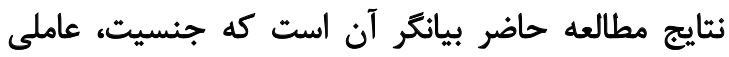

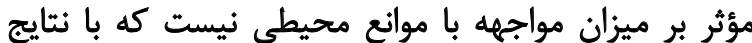

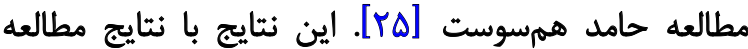

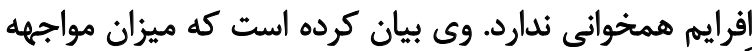

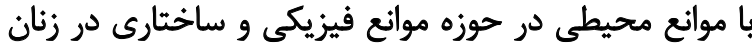

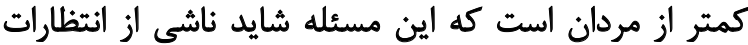

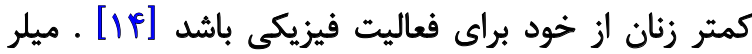

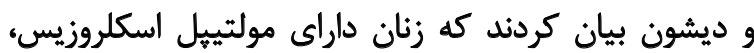

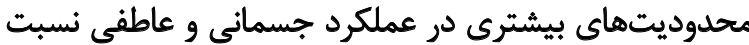

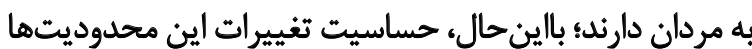

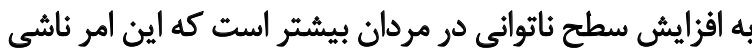

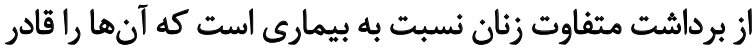

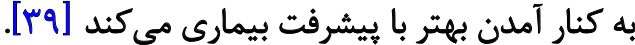
از ديغر نتايج مطالعه حاضر، وجود تفاوت معنادار در ميزان

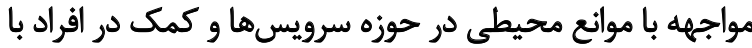

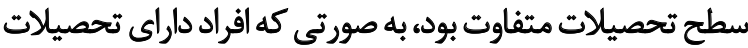

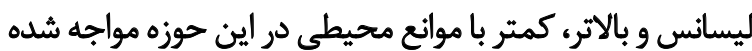

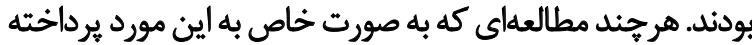

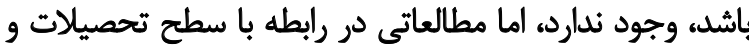

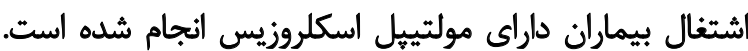

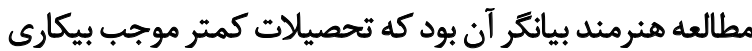

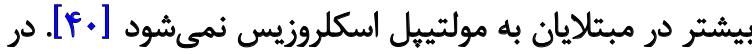

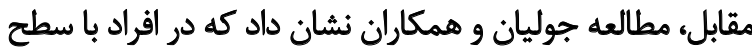

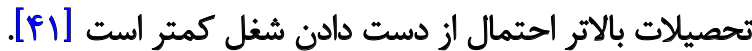

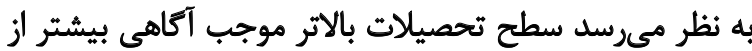

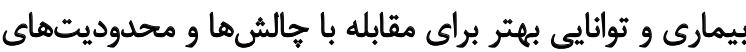
حركتى ناشى از بيمارىهاى مزمن ماني ماند مولتييل اسكلروزيس

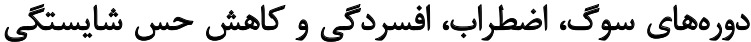

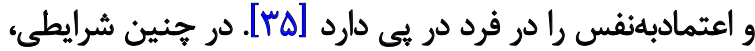

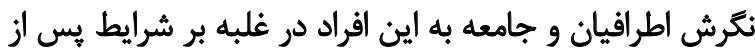

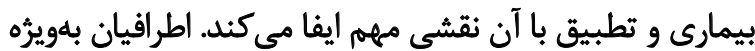

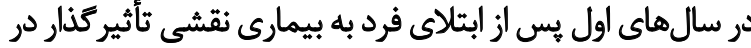

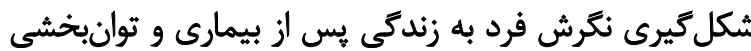

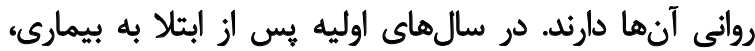

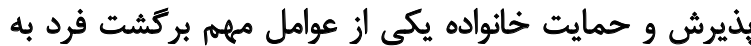

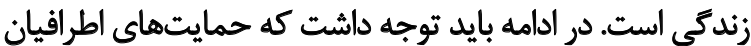

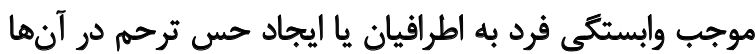

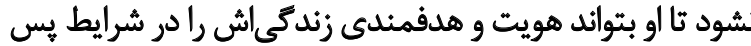

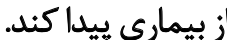

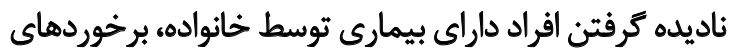

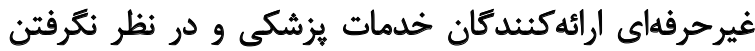

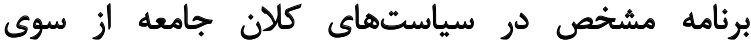

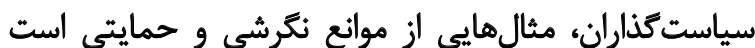

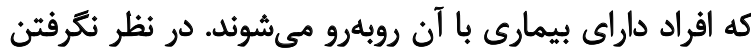

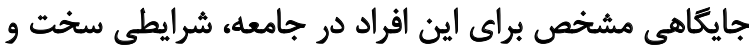

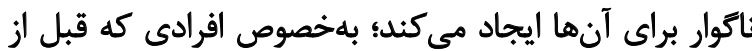

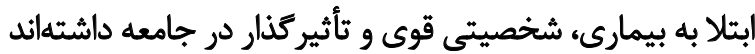

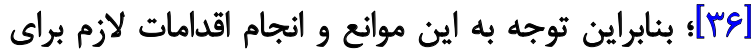

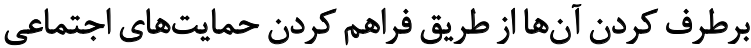

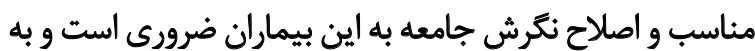

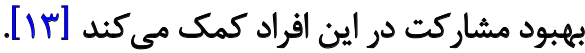

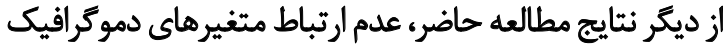

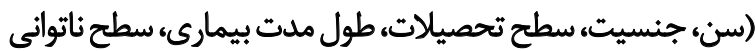

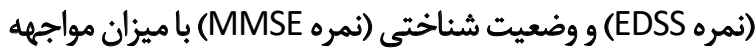

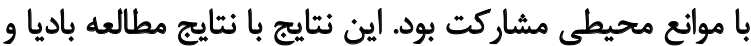

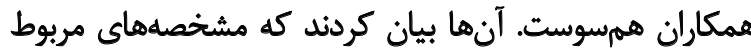

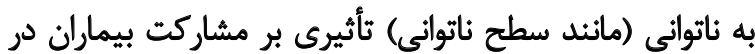

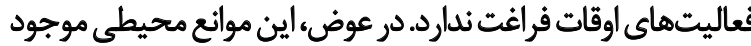

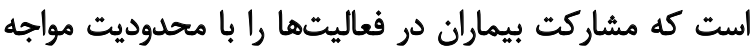

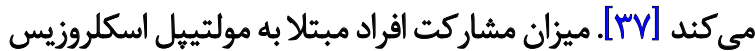

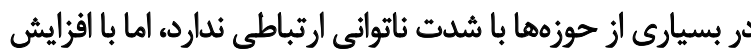

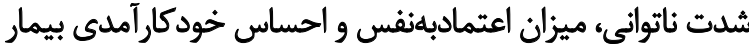

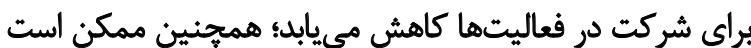

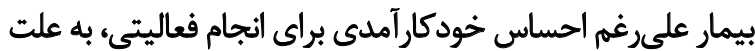

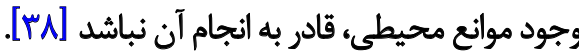

ديكر نتايج مطالعه حاضر در ارتباط با متغيرهاي دموكرافيك

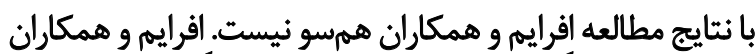

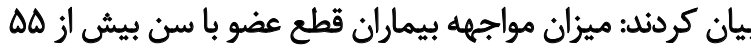

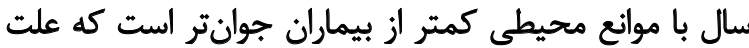
آن ممكن است توانايى بيشتر افراد مسن در انيمان جوانتر انطباق با ناتوانى عات 
مي شود و سطح مشاركت افراد در انجام فعاليتهاي روزمره را تحت تأثير قرار مى دهد مثاركت [FY]

نويسندكان مقاله تشكر و قدردانى خود را از مركز تحقيقات

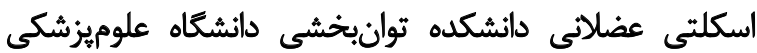

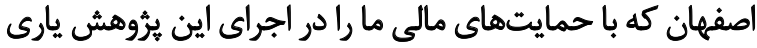

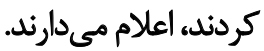

\section{تئيجنيرى}

در بررسى موانع محيطى اثركذار بر مشاركت افراد مبتلا به دانه

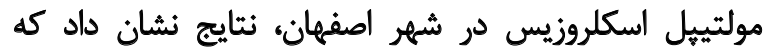

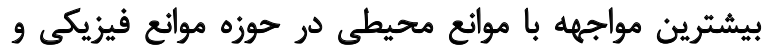

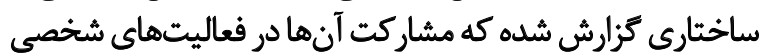
و اجتماعى را محدود كرده است كارداركت

با توجه به شيوع بالاى مولتييل اسكلروزيس در ايران و وانيان

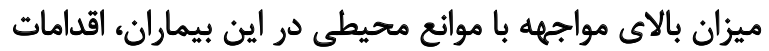

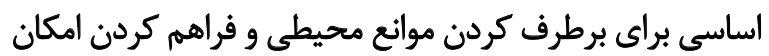

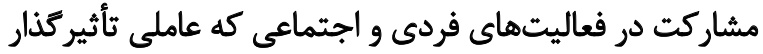

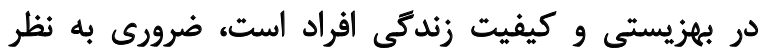

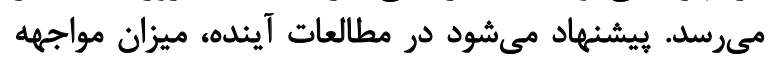

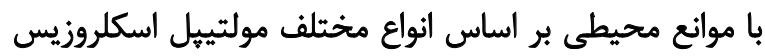

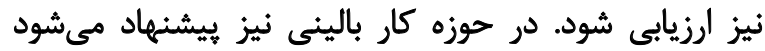

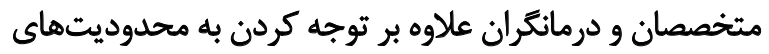

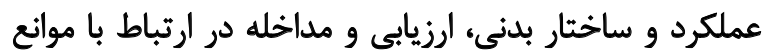
محيطى را نيز مد نظر قرار دهند.

مهمثرين محدوديت مطالعه حاضر آن است كه برسشنامئه

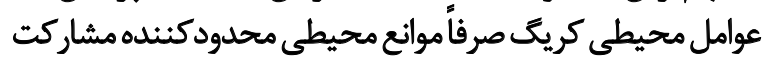
رامىسنجد و عوامل محيطى تسهيل كنينده رابر مرسى نمى كند.

مالاحظات اخلاقي

\section{ييروى أز اصول اخلاق بثوهش}

اصول اخلاقى بروهش براي اين مطالعه به تصويب كميته

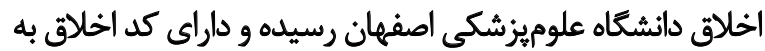

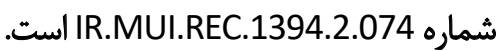

$$
\text { مامي مالي }
$$

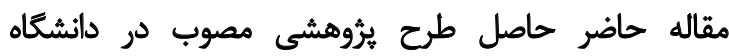

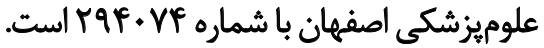

$$
\text { مشاركت نويسندكَان }
$$

تمامى نويسندكان، در نتارش اين مقاله مشاركت داشتهاند.

$$
\text { تعارض منافع }
$$

بنا بر اظهار نويسندكان، اين مقاله تعارض منافع ندارد. 


\section{References}

[1] Umphred D, Lazaro R, Roller M, Burton G. Umphred's neurological rehabilitation. St. Louis: Elsevier/Mosby; 2013.

[2] Zahiri N, Abdollahi I, Nabavi SM, Arab AM. [Explicit motor sequence learning in relapse-remission MS patients (Persian)]. Archives of Rehabilitation. 2013; 14(2):70-6.

[3] Khan F, Pallant JF. Use of international classification of functioning, disability and health (ICF) to describe patient-reported disability in multiple sclerosis and identification of relevant environmental factors. Journal of Rehabilitation Medicine. 2007; 39(1):63-70. [DOI:10.2340/16501977-0002] [PMID]

[4] Mahdizadeh A, Lokzadeh S, Riyahi A, Hosseini SA, Jalili N. [The investigation of factors affecting the gait of patients suffering from multiple sclerosis (Persian)]. Archives of Rehabilitation. 2019; 20(1):64-73. [DOI:10.32598/rj.20.1.64]

[5] Halper J. The evolution of nursing care in multiple sclerosis. International Journal of MS Care. 2000; 2(1):14-22. [DOI:10.7224/1537-2073-2.1.14]

[6] Rosenbaum P, Stewart D. The world health organization international classification of functioning, disability, and health: A model to guide clinical thinking, practice and research in the field of cerebral palsy. Seminars in Pediatric Neurology. 2004; 11(1):5-10. [DOI:10.1016/j.spen.2004.01.002] [PMID]

[7] Jalili N, Nasr Z, Zare R, Sattari M, Hosseini A. [Occupational performance priorities of children with cerebral palsy from the parents' perspective (Persian)]. Archives of Rehabilitation. 2019; 19(4):370-9. [DOI:10.32598/rj.19.4.370]

[8] King G, Lawm M, King S, Rosenbaum P, Kertoy MK, Young NL. A conceptual model of the factors affecting the recreation and leisure participation of children with disabilities. Physical \& Occupational Therapy in Pediatrics. 2003; 23(1):63-90. [DOI:10.1080/J006v23n01_05]

[9] Ewert T, Fuessl M, Cieza A, Andersen C, Chatterji S, Kostanjsek $\mathrm{N}$, et al. Identification of the most common patient problems in patients with chronic conditions using the ICF checklist. Journal of Rehabilitation Medicine. 2004; 36(1):229. [DOI:10.1080/16501960410015362] [PMID]

[10] Hosseini MS, Rassafiani M, Mazdeh M, Haghgoo H, NuraniGHaraborghe S. [Effect of fatigue, depression and cognitive dysfunction on participation in leisurely activities among people with multiple sclerosis (Persian)]. Middle Eastern Journal of Disability Studies. 2017; 7(36):1-7.

[11] Bronfenbrenner U. Environments in developmental perspective: Theoretical and operational models. In: Friedman SL, Wachs TD, editors. Measuring Environment Across the Life Span: Emerging Methods and Concepts. Washington, D.C.: American Psychological Association; 1999. pp. 3-28. [DOI:10.1037/10317-001]

[12] Verdonschot MM, De Witte L, Reichrath E, Buntinx W, Curfs L. Impact of environmental factors on community participation of persons with an intellectual disability: A systematic review. Journal of Intellectual Disability Research. 2009; 53(1):54-64. [DOI:10.1111/j.1365-2788.2008.01128.x] [PMID]
[13] Lexell EM, Lund ML, Iwarsson S. Constantly changing lives: Experiences of people with multiple sclerosis. American Journal of Occupational Therapy. 2009; 63(6):772-81. [DOI:10.5014/ajot.63.6.772] [PMID]

[14] Ephraim PL, MacKenzie EJ, Wegener ST, Dillingham TR, Pezzin LE. Environmental barriers experienced by amputees: The Craig Hospital inventory of environmental factors-short form. Archives of Physical Medicine and Rehabilitation. 2006; 87(3):328-33. [DOI:10.1016/j.apmr.2005.11.010] [PMID]

[15] Law M, Petrenchik T, King G, Hurley P. Perceived environmental barriers to recreational, community, and school participation for children and youth with physical disabilities. Archives of Physical Medicine and Rehabilitation. 2007; 88(12):1636-42. [DOI:10.1016/j.apmr.2007.07.035] [PMID]

[16] Liao LR, Lau RW, Pang MY. Measuring environmental barriers faced by individuals living with stroke: Development and validation of the Chinese version of the Craig Hospital Inventory of Environmental Factors. Journal of Rehabilitation Medicine. 2012; 44(9):740-6. [DOI:10.2340/16501977-1014] [PMID]

[17] Keysor JJ, Jette AM, LaValley MP, Lewis CE, Torner JC, Nevitt $\mathrm{MC}$, et al. Community environmental factors are associated with disability in older adults with functional limitations: The MOST study. The Journals of Gerontology: Series A. 2010; 65A(4):393-9. [DOI:10.1093/gerona/glp182] [PMID] [PMCID]

[18] Rimmer JH, Riley B, Wang E, Rauworth A, Jurkowski J. Physical activity participation among persons with disabilities: Barriers and facilitators. American Journal of Preventive Medicine. 2004; 26(5):419-25. [DOI:10.1016/j.amepre.2004.02.002] [PMID]

[19] Ramirez A, Farmer GC, Grant D, Papachristou T. Disability and preventive cancer screening: Results from the 2001 California health interview survey. American Journal of Public Health. 2005; 95(11):2057-64. [DOI:10.2105/ AJPH.2005.066118] [PMID] [PMCID]

[20] Sahraian MA, Khorramnia S, Ebrahim MM, Moinfar Z, Lotfi J, Pakdaman H. Multiple sclerosis in Iran: A demographic study of 8,000 patients and changes over time. European Neurology. 2010; 64(6):331-6. [DOI:10.1159/000321649] [PMID]

[21] Etemadifar M, Maghzi AH. Sharp increase in the incidence and prevalence of multiple sclerosis in Isfahan, Iran. Multiple Sclerosis Journal. 2011; 17(8):1022-7. [DOI:10.1177/1352458511401460] [PMID]

[22] Foroughan M, Jafari Z, Shirin Bayan P, Ghaem Magham Farahani Z, Rahgozar M. [Validation of Mini- Mental State Examination (MMSE) in the elderly population of Tehran (Persian)]. Advances in Cognitive Science. 2008; 10(2):29-37.

[23] Atashzadeh F, Shiri H, Moshtaqe Esheqi Z, Saniei M. [Effect of exercise training on activity of daily living in women with multiple sclerosis in Iranian multiple sclerosis society (Persian)]. Journal of Rafsanjan University of Medical Sciences. 2003; 2(3-4):164-71.

[24] Nobakht Z, Rassafiani M, Rezasoltani P. Validity and reliability of Persian version of Craig Hospital Inventory of Environmental Factors (CHIEF) in children with cerebral palsy. Iranian Rehabilitation Journal. 2011; 9(1):3-10. 
[25] Hamed R. Environmental factors affecting the daily functioning of Jordanian individuals with multiple sclerosis. International Journal of MS Care. 2012; 14(4):169-78. [DOI:10.7224/1537-2073-14.4.169] [PMID] [PMCID]

[26] Zhang L, Yan T, You L, Li K. Barriers to activity and participation for stroke survivors in rural China. Archives of Physical Medicine and Rehabilitation. 2015; 96(7):1222-8. [DOI:10.1016/j.apmr.2015.01.024] [PMID]

[27] Ebadi A, Hashemi Sheykh Shabani E, Ghasemzadeh R, Latifi SM, Jafari GA. [Identifying the barriers to employment of people with physical disabilities and its implications for rehabilitation policy makers (Persian)]. Middle Eastern Journal of Disability Studies. 2013; 3(2):9-22.

[28] Rolfe D, Yoshida K, Renwick R, Bailey C. Balancing safety and autonomy: Structural and social barriers affecting the exercise participation of women with disabilities in community recreation and fitness facilities. Qualitative Research in Sport, Exercise and Health. 2012; 4(2):265-83. [DOI:10.1080 /2159676X.2012.685099]

[29] Mortenson WB, Miller WC, Backman CL, Oliffe JL. Association between mobility, participation, and wheelchair-related factors in long-term care residents who use wheelchairs as their primary means of mobility. Journal of the American Geriatrics Society. 2012; 60(7):1310-5. [DOI:10.1111/j.15325415.2012.04038.x] [PMID] [PMCID]

[30] Cardol M, de Jong BA, van den Bos GAM, Beelen A, de Groot IJM, de Haan RJ. Beyond disability: Perceived participation in people with a chronic disabling condition. Clinical Rehabilitation. 2002; 16(1):27-35. [DOI:10.1191/0269215502cr464oa] [PMID]

[31] Whiteneck GG, Harrison-Felix CL, Mellick DC, Brooks C, Charlifue SB, Gerhart KA. Quantifying environmental factors: A measure of physical, attitudinal, service, productivity, and policy barriers. Archives of Physical Medicine and Rehabilitation. 2004; 85(8):1324-35. [DOI:10.1016/j. apmr.2003.09.027] [PMID]

[32] Uccelli MM, Specchia C, Battaglia M, Miller D. Factors that influence the employment status of people with multiple sclerosis: A multi-national study. Journal of Neurology. 2009; 256(12):1989-96. [DOI:10.1007/s00415-009-5225-0] [PMID]

[33] Gray DB, Gould M, Bickenbach J. Environmental barriers and disability. Journal of Architectural and Planning Research. 2003; 20(1):29-37.

[34] Keysor JJ, Jette AM, Coster W, Bettger JP, Haley SM. Association of environmental factors with levels of home and community participation in an adult rehabilitation cohort. Archives of Physical Medicine and Rehabilitation. 2006; 87(12):1566-75. [DOI:10.1016/j.apmr.2006.08.347] [PMID]

[35] Goretti B, Portaccio E, Zipoli V, Razzolini L, Amato M. Coping strategies, cognitive impairment, psychological variables and their relationship with quality of life in multiple sclerosis. Neurological Sciences. 2010; 31(2):227-30. [DOI:10.1007/ s10072-010-0372-8] [PMID]

[36] Hosseni-Golafshani Z, Abedi H, Ahmadi F. [Reflection on the experiences of disabled people: The society as a cause of growth or barrier (Persian)]. Journal of Qualitative Research in Health Sciences. 2013; 2(3):248-60.
[37] Badia M, Orgaz BM, Verdugo MA, Ullán AM, Martínez MM. Personal factors and perceived barriers to participation in leisure activities for young and adults with developmental disabilities. Research in Developmental Disabilities. 2011; 32(6):2055-63. [DOI:10.1016/j.ridd.2011.08.007] [PMID]

[38] Yorkston KM, Kuehn CM, Johnson KL, Ehde DM, Jensen MP, Amtmann D. Measuring participation in people living with multiple sclerosis: A comparison of self-reported frequency, importance and self-efficacy. Disability and Rehabilitation. 2008; 30(2):88-97. [DOI:10.1080/09638280701191891] [PMID] [PMCID]

[39] Miller A, Dishon S. Health-related quality of life in multiple sclerosis: The impact of disability, gender and employment status. Quality of Life Research. 2006; 15(2):259-71. [DOI:10.1007/s11136-005-0891-6] [PMID]

[40] Honarmand K, Akbar N, Kou N, Feinstein A. Predicting employment status in multiple sclerosis patients: The utility of the MS functional composite. Journal of Neurology. 2011; 258(2):244-9. [DOI:10.1007/s00415-010-5736-8] [PMID]

[41] Julian L, Vella L, Vollmer T, Hadjimichael O, Mohr DC. Employment in multiple sclerosis. Journal of Neurology. 2008; 255(9):1354-60 [DOI:10.1007/s00415-008-0910-y] [PMID] [PMCID]

[42] Patti F, Pozzilli C, Montanari E, Pappalardo A, Piazza L, Levi $A$, et al. Effects of education level and employment status on HRQoL in early relapsing-remitting multiple sclerosis. Multiple Sclerosis Journal. 2007; 13(6):783-91. [DOI:10.1177/1352458506073511] [PMID] 